\title{
Reduction of rounding noise and lifting steps in non-separable four-dimensional quadruple lifting integer wavelet transform
}

\author{
Fairoza Amira Binti Hamzah ${ }^{1 *}$, Sayaka Minewaki ${ }^{1}$, Taichi Yoshida ${ }^{2}$ and Masahiro Iwahashi ${ }^{1}$
}

\begin{abstract}
The wavelet transform (WT)-based JPEG 2000 is a standard for the compression of digital images that uses a separable lifting structure in which a multidimensional image signal is transformed separately along its horizontal and vertical dimensions. A non-separable three-dimensional (3D) structure is used to minimize the number of lifting steps in existing methods and can reduce the delay between input and output as each process is implemented by cascading in lifting calculation. This structure reduces rounding noise and the number of steps of the lifting scheme in the transform. The non-separable 3D structure in the 5/3-type transform for lossless coding reduces rounding noise, but it increases in the 9/7-type transform for lossy coding in the structure. A combination of 2D and 3D non-separable structures for 4D integer WT has been proposed to solve this problem, but the original filter arrangements need to be preserved to reduce rounding noise. Therefore, in this study, a non-separable 2D structure for the integer implementation of a 4D quadruple lifting WT with a 9/7 filter is proposed. The proposed wavelet transform has the same output signal as the conventional separable structure except for the rounding noise. As the order of the original lifting scheme is preserved, rounding noise in pixels of the decoded image can be significantly reduced, and the upper bounds of quality and lossy decoded 4D medical images can be improved.
\end{abstract}

Keywords: Wavelet, Transform, Lifting, 4D, Rounding, Coding, Quadruple

\section{Introduction}

Discrete cosine transform (DCT)-based digital image signal compression was superseded by the discrete wavelet transform (DWT)-based JPEG 2000 as the standard used to compress digital images [1]. The JPEG 2000 restricts the user's choice to two wavelet transforms-Daubechies $9 / 7$ for lossy compression [2] and the 5/3 LeGall wavelet [3], which has rational coefficients for reversible or lossless compression. The JPEG 2000 also supports arbitrary transform kernels and specifies that they should be implemented by using a lifting scheme [4].

Recent advances in multidimensional image data have enhanced the importance of research on suitable compression methods. Digital multimedia technologies have progressed from one-dimensional (1D) audio signals, and 2D and 3D image signals to $4 \mathrm{D}$ signals. The medical imaging industry

\footnotetext{
*Correspondence: fairozaamira@gmail.com

${ }^{1}$ Department of Electrical, Electronics and Information Engineering, Nagaoka University of Technology, Niigata, Japan

Full list of author information is available at the end of the article
}

has also progressed to a filmless environment where the amount of digital data that need to be managed presents a significant challenge. Four-dimensional images are increasingly being collected and used in clinical and research applications, such as 4D magnetic resonance imaging (MRI), computed tomography $(\mathrm{CT})$, ultrasound, and functional MRI. Four-dimensional medical images have had a significant influence on the diagnosis of diseases and surgical planning [5]. An image slice resolution of $512 \times 512$ has been the minimum standard, but nowadays, state-of-the-art scanning systems can output image slices at spatial resolutions of $1024 \times 1024$ or more at increasing pixel bit depths [6]. Limitations on storage space and transmission bandwidth, on the other hand, and the growing size of medical image datasets, on the other, have spurred research on the design of ad hoc tools. The increasing demand for efficiently storing and transmitting digital medical datasets has triggered investigations into multidimensional and dynamic image compression. Thus, a number of studies have examined the compression of 4D medical images, such as [7-9]. 
By adopting the Joint Photographic Experts Group (JPEG) international standard, a class of separable 2D WT has been broadly developed for various applications designed to efficiently compress digital still images. As its transfer function is composed of the product of a 1D transfer function in two spatial dimensions, it can inherit the legacy of previously designed 1D structures suitable for hardware implementation $[10,11]$. It can also feature regularity and low sensitivity to various kinds of noise [12]. Non-separable structures have been primarily introduced to enhance the accuracy of prediction by adapting to the local context of neighboring pixels $[13,14]$. Furthermore, several studies on reducing hardware complexity by introducing parallel processing to image coding were reviewed in [15] and a parallelization of the 2D fast wavelet transform was proposed in [16]. Directionality has recently been utilized in a generalized poly-phase representation [17-19] with the aim of designing adaptive high-pass filters of wavelet transforms.

A new class of non-separable 2D structures has been reported in [20-22], where the transfer function can be expressed as a product of four 1D functions. The transform based on this structure is compatible with the separable transform. The non-separable structure is not a cascade of instances of 1D signal processing in a 1D structure, but requires multidimensional memory access. This structure can reduce the total number of steps of the lifting scheme and the rounding operations therein.

Various types of wavelet transforms have been reported to analyze the geometry of 4D images [23], 4D hyperspectral images [24], 4D medical volumetric data [8, 9], 4D light field data [25], and 4D color images [26]. However, most of them use the separable 4D WT that contains a large amount of rounding noise. Therefore, a non-separable 3D integer WT was proposed in [27] to overcome its limitations. Unfortunately, this was limited to a double-lifting integer WT with a $5 / 3$ filter especially applied for lossless coding. A non-separable quadruple 3D WT with a 9/7 filter was subsequently proposed in [28] for lossy coding. Nevertheless, unlike in the double-lifting WT, the variance of rounding noise increased in the quadruple lifting WT even though the number of lifting steps decreased. Rounding noise in the transform can reduce the efficiency of the lossy coding structure. This paper is the first to use a nonseparable 4D quadruple lifting integer WT with the aim of reducing rounding noise inside the transform as well as improving its coding performance. Note that a part of this paper was presented in [29].

This paper focuses on lossy coding of 4D signals using the 9/7-type transform based on the quadruple lifting steps, and a reduction in rounding noise in the integer implementation of the transform is affected. As a lifting step needs to wait for the results of calculations from the previous lifting step, many sequential lifting steps incur a long delay between input and output. The real numbers assumed as signal values inside the transform are rounded into finite-length rational numbers. Shorter lengths imply lower computational load but more rounding noise. The space needed for memory storage can be reduced in a tradeoff with rounding noise [30].

This paper proposes a non-separable 2D quadruple lifting structure for 4D input signals to deal with the problem of degradation in image quality due to integer implementation. It has the advantage that its output signals, apart from rounding noise, are identical to those of a conventional transform the transfer function of which is a product of $161 \mathrm{D}$ transfer functions. Unlike the prevalent 3D quadruple lifting structure, the order of lifting steps in the original separable $4 \mathrm{D}$ transform is preserved. Thus, the total rounding noise is reduced even though the total number of rounding operators remains the same as in prevalent methods. Experiments confirmed that the total rounding noise observed in each frequency band of the decoded images was significantly smaller. The upper bounds of the quality-decoded images in lossy coding mode also improved.

The remainder of this paper is organized as follows: Section 2 introduces the two types of WT and the lifting structure in 1D WT. This structure is extended to the 4D case in Section 3, where the separable 4D structure is presented as "existing I" method. The non-separable structure is introduced in Section 3.2. It uses a 3D structure for 4D WT and is referred to as "existing II" method. In Section 4, the proposed methods are introduced and compared with the existing methods. They are combinations of non-separable $2 \mathrm{D}$ and $3 \mathrm{D}$ structures, called the "proposed I method," and a nonseparable 2D structure called "proposed II" method. All methods are experimentally compared in terms of various aspects of six input signals in Section 5. The conclusions of this paper are detailed in Section 6.

\section{Wavelet transform}

Figure 1 shows the forward and backward transforms of the integer WT developed for the 5/3-type transform of the lossless coding of a discrete 1D signal in JPEG 2000 [31]. Figure 1a shows the forward transform of the integer WT and Fig. 1b shows its backward transform. It is composed of two lifting steps. The input signal $X$ is down-sampled and fed into the forward transform, which is transformed into a low-frequency band signal, $Y_{\mathrm{L}}$, and a high-frequency band signal $Y_{\mathrm{H}} \cdot A_{1}$ and $A_{2}$ are the coefficients of the filter bank, and $Y_{\mathrm{L}}$ and $Y_{\mathrm{H}}$ are coded with an entropy encoder to generate a bit stream for storage and communication. The band signals are then decoded and inversely transformed to obtain the reconstructed signal, $X$.

Figure 2 shows a 9/7-type transform developed for lossy coding. Two more lifting steps and scaling with a constant $k$ are added in this type. This paper focuses on the 9/7- 


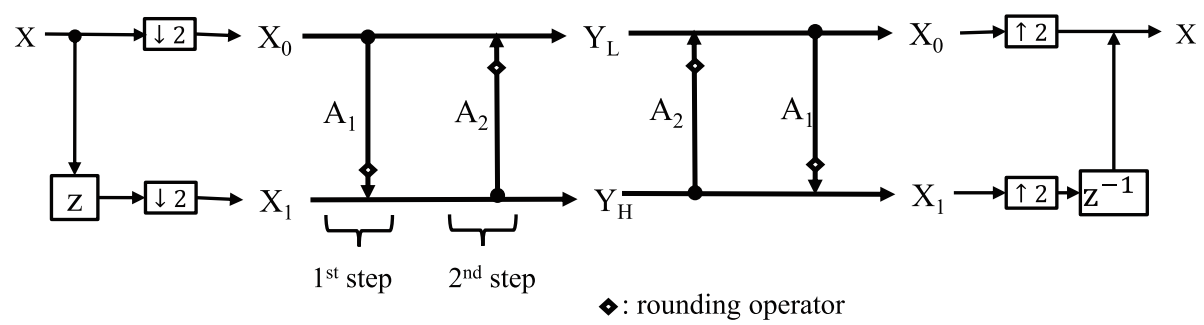

(a) Forward transform

(b) Backward transform

Fig. 1 The 1D integer WT for 5/3-type transform. a Forward transform. b Backward transform

type transform for lossy coding of 4D signals. A problem related to the integer implementation of the transform is addressed here.

In detail, the input signal $x(n), n=0,1, \ldots, N-1$ is divided into two groups $x_{0}(m)$ and $x_{1}(m), m=0,1, \cdots, M-1$, $M=N / 2$. It is expressed with the $z$ transform as

$$
X_{c}(z)=\downarrow 2\left[z^{c} X(z)\right], \quad c \in\{0,1\},
$$

for

$$
\downarrow 2[X(z)]=\frac{1}{Q} \sum_{p=0}^{Q-1} X\left(z^{\frac{1}{Q}} W_{Q}^{p}\right), \quad W_{Q}=e^{\frac{j 2 \pi}{Q}},
$$

where $Q=2$ and

$$
X(z)=\sum_{n=0}^{N-1} x(n) z^{-n}
$$

Secondly, the first lifting step is applied as

$$
X_{1}^{(1)}(z)=X_{1}(z)+R\left[A_{1}(z) X_{0}(z)\right]
$$

and the second lifting is applied as

$$
X_{0}^{(2)}(z)=X_{0}(z)+R\left[A_{2}(z) X_{1}^{(1)}(z)\right]
$$

where $A_{1}(z)$ and $A_{2}(z)$ are filters given as

$$
\left[\begin{array}{l}
A_{1}(z) \\
A_{2}(z)
\end{array}\right]=\left[\begin{array}{l}
h_{1}\left(1+z^{-1}\right) \\
h_{2}\left(1+z^{-1}\right)
\end{array}\right]
$$

Finally, the frequency band signals are generated as

$$
\left[\begin{array}{c}
Y_{L}(z) \\
Y_{H}(z)
\end{array}\right]=\left[\begin{array}{c}
X_{0}^{(2)} \\
X_{1}^{(1)}
\end{array}\right]
$$

for

$$
Y_{b}(z)=\sum_{m=0}^{M-1} y_{b}(m) z^{-m}, \quad b \in\{L, H\},
$$

Note that $R[$ ] denotes the rounding operator that truncates a pixel value in real numbers to an integer. Calculation in a lifting step starts after the calculation results of the previous have been obtained. The greater the number of lifting steps, the higher the latency (or delay). Therefore, the authors reduce the total numbers of lifting steps and rounding operators in the 4D integer WT in the 9/7-type transform. Note that this paper focuses on reducing rounding noise in the transform to increase the coding performance for $4 \mathrm{D}$ data in lossy mode.

In $5 / 3$ type transform, the coefficient values are given as

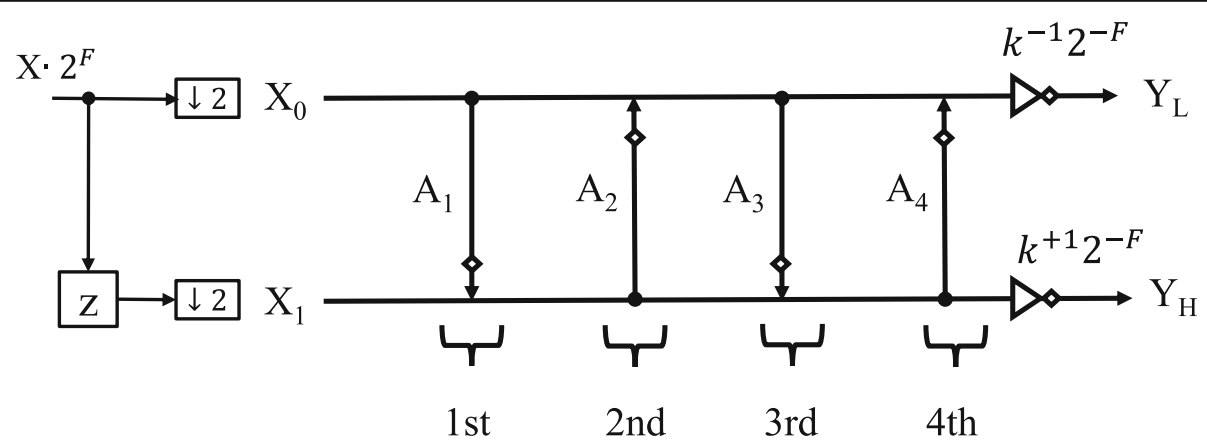

Fig. 2 The integer WT of the 9/7-type transform for lossy coding 


$$
\left[\begin{array}{ccc}
h_{1} & h_{3} & k^{-1} \\
h_{2} & h_{4} & k
\end{array}\right]=\left[\begin{array}{ccc}
-\frac{1}{2} & 0 & 1 \\
\frac{1}{4} & 0 & 1
\end{array}\right],
$$

Lossless reconstruction can be guaranteed with the scaling factors, $k^{-1}$ and $k$ are 1.

The 9/7 type transform has two more lifting steps and scaling. Namely, the third lifting step

$$
X_{1}^{(3)}(z)=X_{1}^{(1)}(z)+R\left[A_{3}(z) X_{0}^{(2)}(z)\right]
$$

and the fourth lifting is applied as

$$
X_{0}^{(4)}(z)=X_{0}^{(2)}(z)+R\left[A_{4}(z) X_{1}^{(3)}(z)\right]
$$

where $A_{3}(z)$ and $A_{4}(z)$ are filters given as

$$
\left[\begin{array}{l}
A_{3}(z) \\
A_{4}(z)
\end{array}\right]=\left[\begin{array}{l}
h_{3}\left(1+z^{-1}\right) \\
h_{4}\left(1+z^{-1}\right)
\end{array}\right]
$$

In 9/7 type transform, the coefficient values are given as

$$
\left\{\begin{array}{c}
h_{1}=-1.586134342059924 \\
h_{2}=-0.052980118572961 \\
h_{3}=0.882911075530934 \\
h_{4}=0.443506852043971 \\
k=1.230174104914001
\end{array}\right.
$$

Finally, the frequency band signals are generated with scaling as

$$
\left[\begin{array}{c}
Y_{L}(z) \\
Y_{H}(z)
\end{array}\right]=\left[\begin{array}{l}
R\left[k^{-1} 2^{-F} X_{0}^{(4)}\right] \\
R\left[k^{+1} 2^{-F} X_{1}^{(3)}\right]
\end{array}\right],
$$

Note that the input signal is scaled with $2^{F}$ beforehand as shown in Fig. 2. In the integer implementation, $F$ is set as a positive number. The smaller the $F$ is, the shorter the bit depth of signals inside the transform will be.

\section{Existing methods}

\subsection{Separable 4D structure (existing I)}

Figure 4 shows the 9/7-type separable 4D integer WT. In the JPEG 2000 standard, the $1 \mathrm{D}$ processing shown in Fig. 2 is applied to a 4D signal along the $x, y, z$, and $t$ dimensions, where $x$ and $y$ denote two spatial dimensions within a slice, $z$ denotes the third spatial dimension within a volume, and $t$ denotes the fourth, temporal, dimension. However, the separable 4D structure increases the number of rounding operators in the transform. This structure has 192 rounding operators.

For a 4D input signal $X(\mathrm{z})$, the transform splits the input signal into 16 channels, $\mathrm{X}_{0000}, \mathrm{X}_{0001}, \mathrm{X}_{0010}, \mathrm{X}_{0011}, \mathrm{X}_{0100}$, $\mathrm{X}_{0101}, \mathrm{X}_{0110}, \mathrm{X}_{0111}, \mathrm{X}_{1000}, \mathrm{X}_{1001}, \mathrm{X}_{1010}, \mathrm{X}_{1011}, \mathrm{X}_{1100}, \mathrm{X}_{1101}$, $\mathrm{X}_{1110}$, and $\mathrm{X}_{1111}$ as shown in Fig. 3. It is denoted as

$$
\left[\begin{array}{c}
X_{0000}(\mathbf{z}) \\
X_{0010}(\mathbf{z}) \\
X_{0100}(\mathbf{z}) \\
X_{0011}(\mathbf{z}) \\
\vdots \\
X_{1110}(\mathbf{z}) \\
X_{1111}(\mathbf{z})
\end{array}\right]=\left[\begin{array}{c}
\downarrow 2_{D}\left[\left[\begin{array}{c}
1 \\
z_{D}
\end{array}\right] W_{1}(\mathbf{z})\right] \\
\downarrow 2_{D}\left[\left[\begin{array}{c}
1 \\
z_{D}
\end{array}\right] W_{2}(\mathbf{z})\right] \\
\vdots \\
\downarrow 2_{D}\left[\left[\begin{array}{c}
1 \\
z_{D}
\end{array}\right] W_{8}(\mathbf{z})\right]
\end{array}\right]
$$

where

$$
\begin{aligned}
& {\left[\begin{array}{l}
W_{1}(\mathbf{z}) \\
W_{2}(\mathbf{z}) \\
W_{3}(\mathbf{z}) \\
W_{4}(\mathbf{z}) \\
W_{5}(\mathbf{z}) \\
W_{6}(\mathbf{z}) \\
W_{7}(\mathbf{z}) \\
W_{8}(\mathbf{z})
\end{array}\right]=\left[\begin{array}{c}
\downarrow 2_{C}\left[\left[\begin{array}{c}
1 \\
z_{C}
\end{array}\right] V_{1}(\mathbf{z})\right] \\
\downarrow 2_{C}\left[\left[\begin{array}{c}
1 \\
z_{C}
\end{array}\right] V_{2}(\mathbf{z})\right] \\
\downarrow 2_{C}\left[\left[\begin{array}{c}
1 \\
z_{C}
\end{array}\right] V_{3}(\mathbf{z})\right] \\
\downarrow 2_{C}\left[\left[\begin{array}{c}
1 \\
z_{C}
\end{array}\right] V_{4}(\mathbf{z})\right]
\end{array}\right],} \\
& {\left[\begin{array}{l}
V_{1}(\mathbf{z}) \\
V_{2}(\mathbf{z}) \\
V_{3}(\mathbf{z}) \\
V_{4}(\mathbf{z})
\end{array}\right]=\left[\begin{array}{l}
\downarrow 2_{B}\left[\left[\begin{array}{c}
1 \\
z_{B}
\end{array}\right] P_{1}(\mathbf{z})\right] \\
\downarrow 2_{B}\left[\left[\begin{array}{c}
1 \\
z_{B}
\end{array}\right] P_{2}(\mathbf{z})\right]
\end{array}\right]} \\
& {\left[\begin{array}{l}
P_{1}(\mathbf{z}) \\
P_{2}(\mathbf{z})
\end{array}\right]=\downarrow 2_{A}\left[\left[\begin{array}{c}
1 \\
z_{A}
\end{array}\right] X(\mathbf{z})\right]}
\end{aligned}
$$

and

$$
\left[\begin{array}{l}
\downarrow 2_{A}[X(\mathbf{z})] \\
\downarrow 2_{B}[X(\mathbf{z})] \\
\downarrow 2_{C}[X(\mathbf{z})] \\
\downarrow 2_{D}[X(\mathbf{z})]
\end{array}\right]=\left[\begin{array}{c}
\frac{1}{Q} \sum_{p=0}^{Q-1} X\left(z_{A}^{1 / Q} \cdot W_{Q}^{p}, z_{B}, z_{C}, z_{D}\right) \\
\frac{1}{Q} \sum_{p=0}^{Q-1} X\left(z_{A}, z_{B}^{1 / Q} \cdot W_{Q}^{p}, z_{C}, z_{D}\right) \\
\frac{1}{Q} \sum_{p=0}^{Q-1} X\left(z_{A}, z_{B}, z_{C}^{1 / Q} \cdot W_{Q}^{p}, z_{D}\right) \\
\frac{1}{Q} \sum_{p=0}^{Q-1} X\left(z_{A}, z_{B}, z_{C}, z_{D}^{1 / Q} \cdot W_{Q}^{p}\right)
\end{array}\right] \cdot 2^{F},
$$

for

$$
\begin{gathered}
X(\mathbf{z})=\sum_{n_{1}=0}^{N_{1}-1} \sum_{n_{2}=0}^{N_{2}-1} \sum_{n_{3}=0}^{N_{3}-1} \sum_{n_{4}=0}^{N_{4}-1} \\
X(\mathbf{n}) z_{A}^{-n_{1}} z_{B}^{-n_{2}} z_{C}^{-n_{4}} z_{D}^{-n_{4}},
\end{gathered}
$$

where $\mathbf{z}=\left(z_{\mathrm{A}}, z_{\mathrm{B}}, z_{\mathrm{C}}, z_{\mathrm{D}}\right)$ and $\mathbf{n}=\left(n_{1}, n_{2}, n_{3}, n_{4}\right)$.

In JPEG 2000 standard, applying the 1st, 2nd, 3rd, and 4th lifting steps in the spatial dimension, $x$ with

$$
\left[\begin{array}{ll}
A_{1}(\mathbf{z}) & A_{3}(\mathbf{z}) \\
A_{2}(\mathbf{z}) & A_{4}(\mathbf{z})
\end{array}\right]=\left[\begin{array}{ll}
h_{1}\left(1+z_{A}^{+1}\right) & h_{3}\left(1+z_{A}^{+1}\right) \\
h_{2}\left(1+z_{A}^{-1}\right) & h_{4}\left(1+z_{A}^{-1}\right)
\end{array}\right]
$$

and the 5th, 6th, 7th, and 8th lifting steps in the spatial dimension, $y$ with 


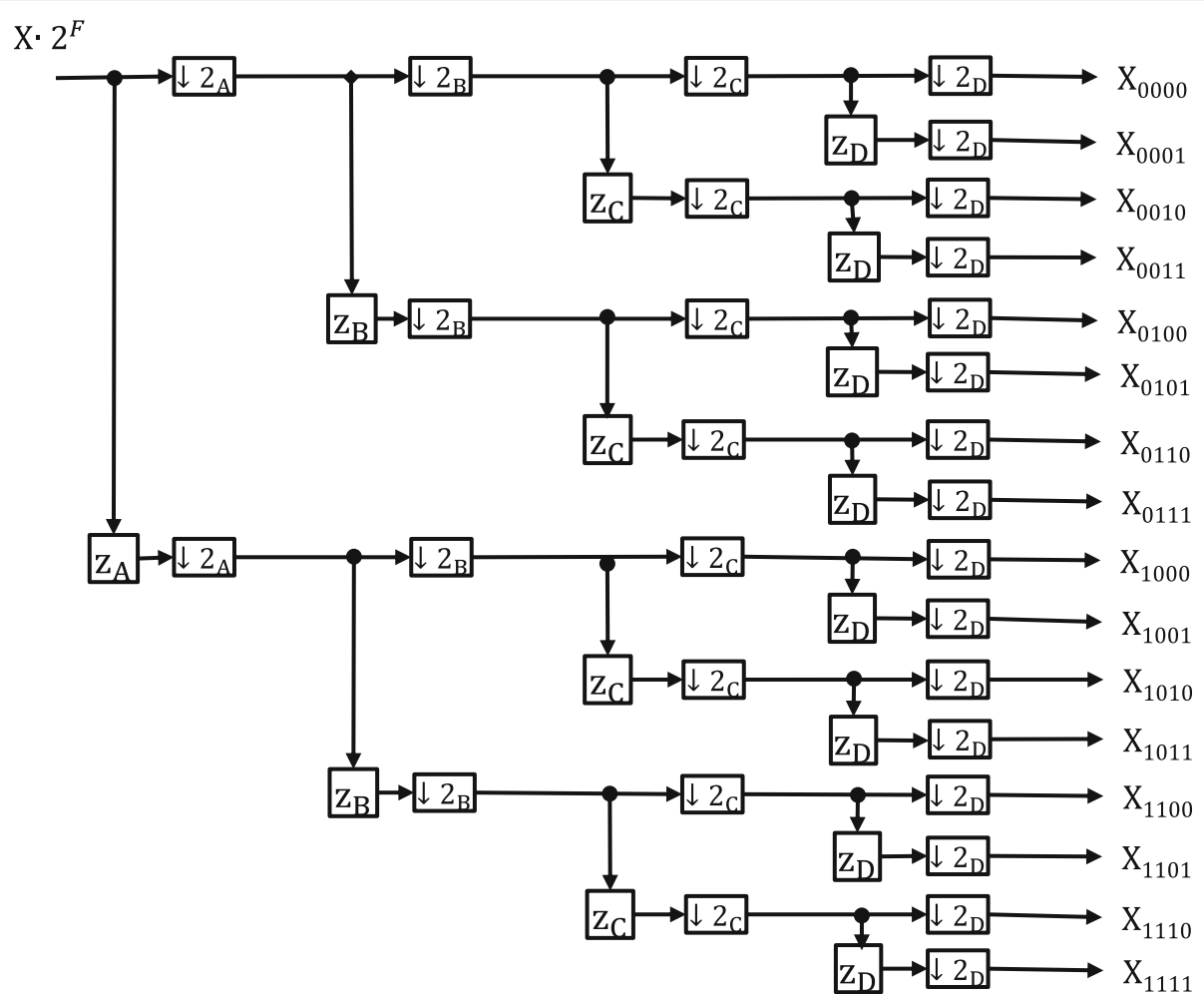

Fig. 3 Decomposition of a 4D signal

$$
\left[\begin{array}{ll}
B_{1}(\mathbf{z}) & B_{3}(\mathbf{z}) \\
B_{2}(\mathbf{z}) & B_{4}(\mathbf{z})
\end{array}\right]=\left[\begin{array}{ll}
h_{1}\left(1+z_{B}^{+1}\right) & h_{3}\left(1+z_{B}^{+1}\right) \\
h_{2}\left(1+z_{B}^{-1}\right) & h_{4}\left(1+z_{B}^{-1}\right)
\end{array}\right],
$$

and the 9th, 10th, 11th, and 12th lifting steps in the spatial dimension, $z$ with

$$
\left[\begin{array}{ll}
C_{1}(\mathbf{z}) & C_{3}(\mathbf{z}) \\
C_{2}(\mathbf{z}) & C_{4}(\mathbf{z})
\end{array}\right]=\left[\begin{array}{ll}
h_{1}\left(1+z_{C}^{+1}\right) & h_{3}\left(1+z_{C}^{+1}\right) \\
h_{2}\left(1+z_{C}^{-1}\right) & h_{4}\left(1+z_{C}^{-1}\right)
\end{array}\right],
$$

and the 13th, 14th, 15th, and 16th lifting steps in the temporal dimension, $t$ with

$$
\left[\begin{array}{ll}
D_{1}(\mathbf{z}) & D_{3}(\mathbf{z}) \\
D_{2}(\mathbf{z}) & D_{4}(\mathbf{z})
\end{array}\right]=\left[\begin{array}{cc}
h_{1}\left(1+z_{D}^{+1}\right) & h_{3}\left(1+z_{D}^{+1}\right) \\
h_{2}\left(1+z_{D}^{-1}\right) & h_{4}\left(1+z_{D}^{-1}\right)
\end{array}\right],
$$

to the channel signals in (7), the transform outputs sixteen frequency band signals $Y_{L L L L}(\mathbf{z}), Y_{L L L H}(\mathbf{z})$, $Y_{L L H L}(\mathbf{z}), \quad Y_{L L H H}(\mathbf{z}), \quad Y_{L H L L}(\mathbf{z}), \quad Y_{L H L H}(\mathbf{z}), \quad Y_{L H H L}(\mathbf{z})$, $Y_{L H H H}(\mathbf{z}), \quad Y_{H L L L}(\mathbf{z}), \quad Y_{H L L H}(\mathbf{z}), \quad Y_{H L H L}(\mathbf{z}), Y_{H L H H}(\mathbf{z})$, $Y_{H H L L}(\mathbf{z}), Y_{H H L H}(\mathbf{z}), Y_{H H H L}(\mathbf{z})$, and $Y_{H H H H}(\mathbf{z})$ as illustrated in Fig. 4. This is referred to as a separable structure. As it has a large number of rounding operators, there is a large volume of rounding noise in the transform. A non-separable 3D structure was thus proposed in [27]. However, when used for a 4D signal, the rounding noise in it significantly increases compared with that in a separable 4D structure. Thus, its coding performance is significantly affected by the rounding noise generated inside it.

\subsection{Non-separable 3D structure (existing II)}

Figure 5 shows the non-separable 3D structure of integer WT for a 4D input signal designed in the 9/7-type transform based on the structure proposed in [28]. In the first to the fourth lifting steps, the 4D input signal, once it is decomposed into 16 channels, is applied to the spatial dimension $x$ as in Eq. (19).

$$
\left[\begin{array}{c}
X_{0000}^{(B)}(\mathbf{z}) \\
X_{0001}^{(B)}(\mathbf{z}) \\
\vdots \\
X_{1111}^{(B)}(\mathbf{z})
\end{array}\right]=\left[\begin{array}{c}
R\left[k^{-1} X_{0000}^{(A)}(\mathbf{z})\right] \\
R\left[k^{-1} X_{0001}^{(A)}(\mathbf{z})\right] \\
\vdots \\
R\left[k^{+1} X_{1111}^{(A)}(\mathbf{z})\right]
\end{array}\right]
$$

Then, from the fifth to the 12th lifting steps, the signals are transformed simultaneously in spatial dimensions $y$ and $z$, and temporal dimension $t$ using the non-separable 3D structure. For instance, the signal in $Y_{\mathrm{LHHH}}$ is produced as 


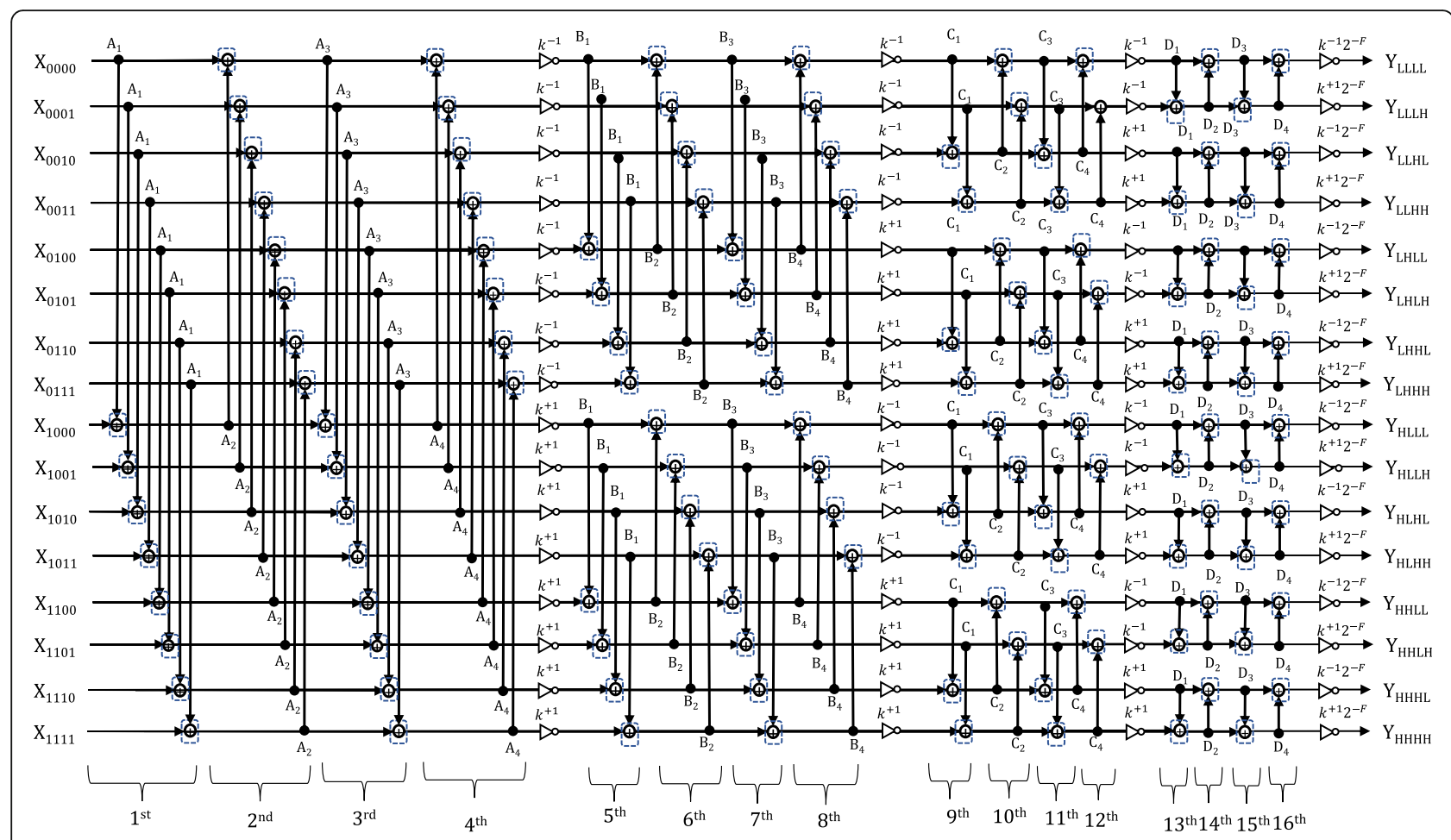

Fig. 4 Separable 4D structure for 9/7-type of transform (existing I)

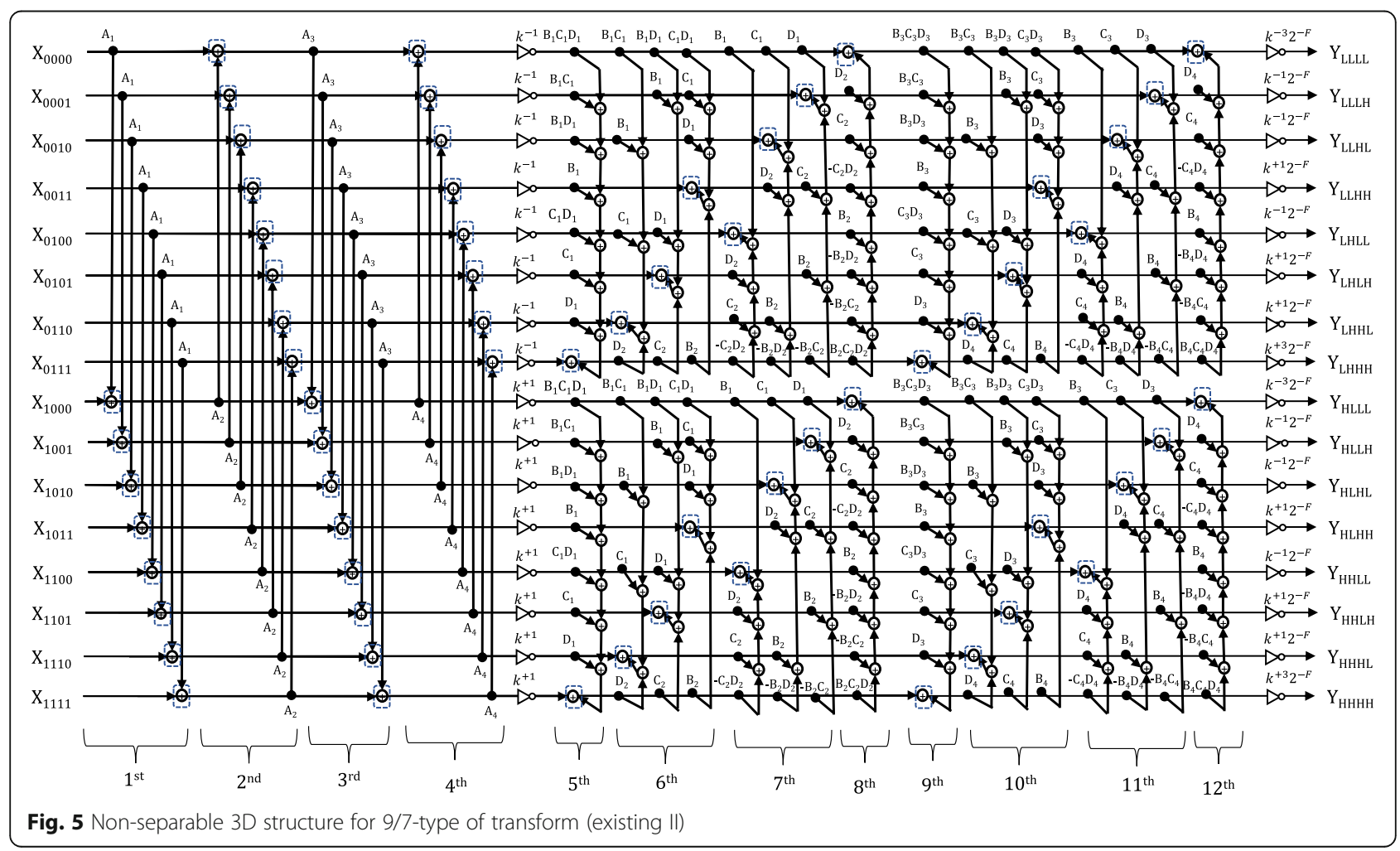




$$
X_{0111}^{(D)}(\mathbf{z})=X_{0111}^{(B)}(\mathbf{z})+R\left[k^{+3} 2^{-F} P_{L H H H}^{(D)}(\mathbf{z})\right]
$$

for

$$
\begin{aligned}
P_{L H H H}^{(D)}= & {\left[\begin{array}{c}
B_{1}(\mathbf{z}) C_{1}(\mathbf{z}) D_{1}(\mathbf{z}) \\
B_{1}(\mathbf{z}) C_{1}(\mathbf{z}) \\
B_{1}(\mathbf{z}) D_{1}(\mathbf{z}) \\
B_{1}(\mathbf{z}) \\
C_{1}(\mathbf{z}) D_{1}(\mathbf{z}) \\
C_{1}(\mathbf{z}) \\
D_{1}(\mathbf{z})
\end{array}\right]^{T}\left[\begin{array}{c}
X_{0000}^{(B)}(\mathbf{z}) \\
X_{001}^{(B)}(\mathbf{z}) \\
X_{010}^{(B)}(\mathbf{z}) \\
X_{0011}^{(B)}(\mathbf{z}) \\
X_{0100}^{(B)}(\mathbf{z}) \\
X_{0101}^{(B)}(\mathbf{z}) \\
X_{0110}^{(B)}(\mathbf{z})
\end{array}\right] } \\
+ & {\left[\begin{array}{c}
B_{3}(\mathbf{z}) C_{3}(\mathbf{z}) D_{3}(\mathbf{z}) \\
B_{3}(\mathbf{z}) C_{3}(\mathbf{z}) \\
B_{3}(\mathbf{z}) D_{3}(\mathbf{z}) \\
B_{3}(\mathbf{z}) \\
C_{3}(\mathbf{z}) D_{3}(\mathbf{z}) \\
C_{3}(\mathbf{z}) \\
D_{3}(\mathbf{z})
\end{array}\right]^{T}\left[\begin{array}{c}
X_{0000}^{(B)}(\mathbf{z}) \\
X_{0001}^{(B)}(\mathbf{z}) \\
X_{010}^{(B)}(\mathbf{z}) \\
X_{0011}^{(B)}(\mathbf{z}) \\
X_{0100}^{(B)}(\mathbf{z}) \\
X_{0101}^{(B)}(\mathbf{z}) \\
X_{0110}^{(B)}(\mathbf{z})
\end{array}\right], }
\end{aligned}
$$

in the fifth lifting step. In this step, a 3D filtering with 3D memory accessing $B_{1}(\mathbf{z}) C_{1}(\mathbf{z}) D_{1}(\mathbf{z})$ is used. In the sixth lifting step, the calculation of $Y_{\mathrm{LHHL}}, Y_{\mathrm{LHLH}}$, and $Y_{\mathrm{LLHH}}$ :

$$
\left[\begin{array}{c}
X_{0110}^{(D)}(\mathbf{z}) \\
X_{0101}^{(D)}(\mathbf{z}) \\
X_{0011}^{(D)}(\mathbf{z})
\end{array}\right]=\left[\begin{array}{c}
X_{0110}^{(B)}(\mathbf{z})+R\left[k^{+1} 2^{-F} P_{L H H L}^{(D)}(\mathbf{z})\right] \\
X_{0101}^{(B)}(\mathbf{z})+R\left[k^{+1} 2^{-F} P_{L H L H}^{(D)}(\mathbf{z})\right] \\
X_{0011}^{(B)}(\mathbf{z})+R\left[k^{+1} 2^{-F} P_{L L H H}^{(D)}(\mathbf{z})\right]
\end{array}\right]
$$

for

$$
\left[\begin{array}{l}
P_{L L H L}^{(D) \prime}(\mathbf{z}) \\
P_{L L H H}^{(D)}(\mathbf{z}) \\
P_{L L H H}^{(D)}(\mathbf{z})
\end{array}\right]=\left[\begin{array}{ccccc}
B_{1}(\mathbf{z}) C_{1}(\mathbf{z}) & 0 & B_{1}(\mathbf{z}) & C_{1}(\mathbf{z}) & D_{2}(\mathbf{z}) \\
B_{1}(\mathbf{z}) D_{1}(\mathbf{z}) & B_{1}(\mathbf{z}) & 0 & D_{1}(\mathbf{z}) & C_{2}(\mathbf{z}) \\
C_{1}(\mathbf{z}) D_{1}(\mathbf{z}) & C_{1}(\mathbf{z}) & D_{1}(\mathbf{z}) & 0 & B_{2}(\mathbf{z})
\end{array}\right]\left[\begin{array}{c}
X_{000}^{(B)}(\mathbf{z}) \\
X_{001}^{(B)}(\mathbf{z}) \\
X_{010}^{(B)}(\mathbf{z}) \\
X_{010(}^{(B)}(\mathbf{z}) \\
X_{0111}^{(B)}(\mathbf{z})
\end{array}\right]
$$

$$
\left[\begin{array}{l}
P_{L L H L}^{(D) \prime \prime}(\mathbf{z}) \\
P_{L L L H}^{(D) \prime}(\mathbf{z}) \\
P_{L L H H}^{(D) ! H}(\mathbf{z})
\end{array}\right]=\left[\begin{array}{ccccc}
B_{3}(\mathbf{z}) C_{3}(\mathbf{z}) & 0 & B_{3}(\mathbf{z}) & C_{3}(\mathbf{z}) & D_{4}(\mathbf{z}) \\
B_{3}(\mathbf{z}) D_{3}(\mathbf{z}) & B_{3}(\mathbf{z}) & 0 & D_{3}(\mathbf{z}) & C_{4}(\mathbf{z}) \\
C_{3}(\mathbf{z}) D_{3}(\mathbf{z}) & C_{3}(\mathbf{z}) & D_{3}(\mathbf{z}) & 0 & B_{4}(\mathbf{z})
\end{array}\right]\left[\begin{array}{c}
X_{0000}^{(B)}(\mathbf{z}) \\
X_{0001}^{(B)}(\mathbf{z}) \\
X_{00101}^{(B)}(\mathbf{z}) \\
X_{0100}^{(B)}(\mathbf{z}) \\
X_{0111}^{(B)}(\mathbf{z})
\end{array}\right],
$$

$$
\left\{\begin{array}{l}
P_{L H H L}^{(D)}(\mathbf{z})=P_{L H H L}^{(D) \prime}(\mathbf{z})+P_{L H H L}^{(D) \prime \prime}(\mathbf{z}) \\
P_{L H L H}^{(D)}(\mathbf{z})=P_{L H L H}^{(D) \prime}(\mathbf{z})+P_{L H L H}^{(D) \prime \prime}(\mathbf{z}) \\
P_{L L H H}^{(D)}(\mathbf{z})=P_{L L H H}^{(D) \prime}(\mathbf{z})+P_{L L H H}^{(D) \prime \prime}(\mathbf{z})
\end{array}\right.
$$

where $R[]$ denotes the rounding operation on a signal value. Similarly, prediction of $X_{1111}, X_{1110}, X_{1101}, X_{1100}$, $X_{1011}, X_{1010}, X_{1001}, X_{1000}, X_{0100}, X_{0010}, X_{0001}$, and $X_{0000}$ are also independent. The total numbers of lifting steps and rounding operators in the non-separable structure were hence reduced from 16 to 12 and 192 to 96 , respectively, comparing with the separable structure in Fig. 4. However, the quality of the decoded image was degraded by the rounding noise inside the transform in its integer implementation. The proposed methods solve this problem as explained below.

\section{Proposed methods}

4.1 Non-separable 2D and 3D structure (proposed I)

To solve the problems of higher rounding noise and degraded quality of lossy coding problems, the nonseparable structure, which combines both $2 \mathrm{D}$ and $3 \mathrm{D}$ structures, is proposed. The total number of rounding operators is thus further reduced from 96 to 72 . Figure 6 illustrates the structure of the proposed I method. Once the $4 \mathrm{D}$ input signal is decomposed into 16 channels, the first, second, and third lifting steps are cascaded by using the non-separable 2D structure, followed by the nonseparable 3D structure and the separable 1D structure. For example, the first lifting step is expressed as

$$
\begin{aligned}
X_{11 c_{1} c_{2}}^{(1)}(\mathbf{z})= & X_{11 c_{1} c_{2}}(\mathbf{z}) \\
& +R\left[A_{1} B_{1} X_{00 c_{1} c_{2}}(\mathbf{z})+A_{1} X_{01 c_{1} c_{2}}(\mathbf{z})+B_{1} X_{10 c_{1} c_{2}}(\mathbf{z})\right], \\
& \text { where } c_{1}, c_{2} \in\{0,1\}
\end{aligned}
$$

Even though this proposed method has fewer rounding operators, the rounding noise in it is still higher than in Existing I method, the separable 4D structure. Therefore, it is necessary to maintain the original structure so that rounding noise in the transform is lower.

\subsection{Non-separable 2D structure (proposed II)}

To lower the rounding noise inside the transform, the non-separable 2D structure for 4D input signals is proposed. Figure 7 shows the proposed II method. Unlike the existing I, existing II, and proposed I methods, it consists of only a non-separable 2D structure. The original order of lifting steps for each dimension is also maintained in this structure. The total number of lifting steps, however, is larger in existing II but smaller than existing I. The total number of rounding operators is smaller in existing I, and the rounding noise is lower, as confirmed in Section 5.

The first and second lifting steps involve 1D structures expressed as

$$
\left\{\begin{array}{c}
X_{1 c_{1} c_{2} c_{3}}^{(1)}(\mathbf{z})=X_{1 c_{1} c_{2} c_{3}}(\mathbf{z})+R\left[A_{1} X_{0 c_{1} c_{2} c_{3}}(\mathbf{z})\right] \\
X_{0 c_{1} c_{2} c_{3}}^{(1)}(\mathbf{z})=X_{0 c_{1} c_{2} c_{3}}(\mathbf{z})+R\left[A_{2} X_{1 c_{2} c_{3}}(\mathbf{z})\right]
\end{array}, \quad \text { where } c_{1}, c_{2}, c_{3} \in\{0,1\},\right.
$$

The third, fourth, and fifth lifting steps consist of nonseparable 2D structures, and the same goes for the fifth to the 11th steps. Finally, the 12th and 13th lifting steps involve the separable 1D structure. For example, the third lifting step is expressed as 

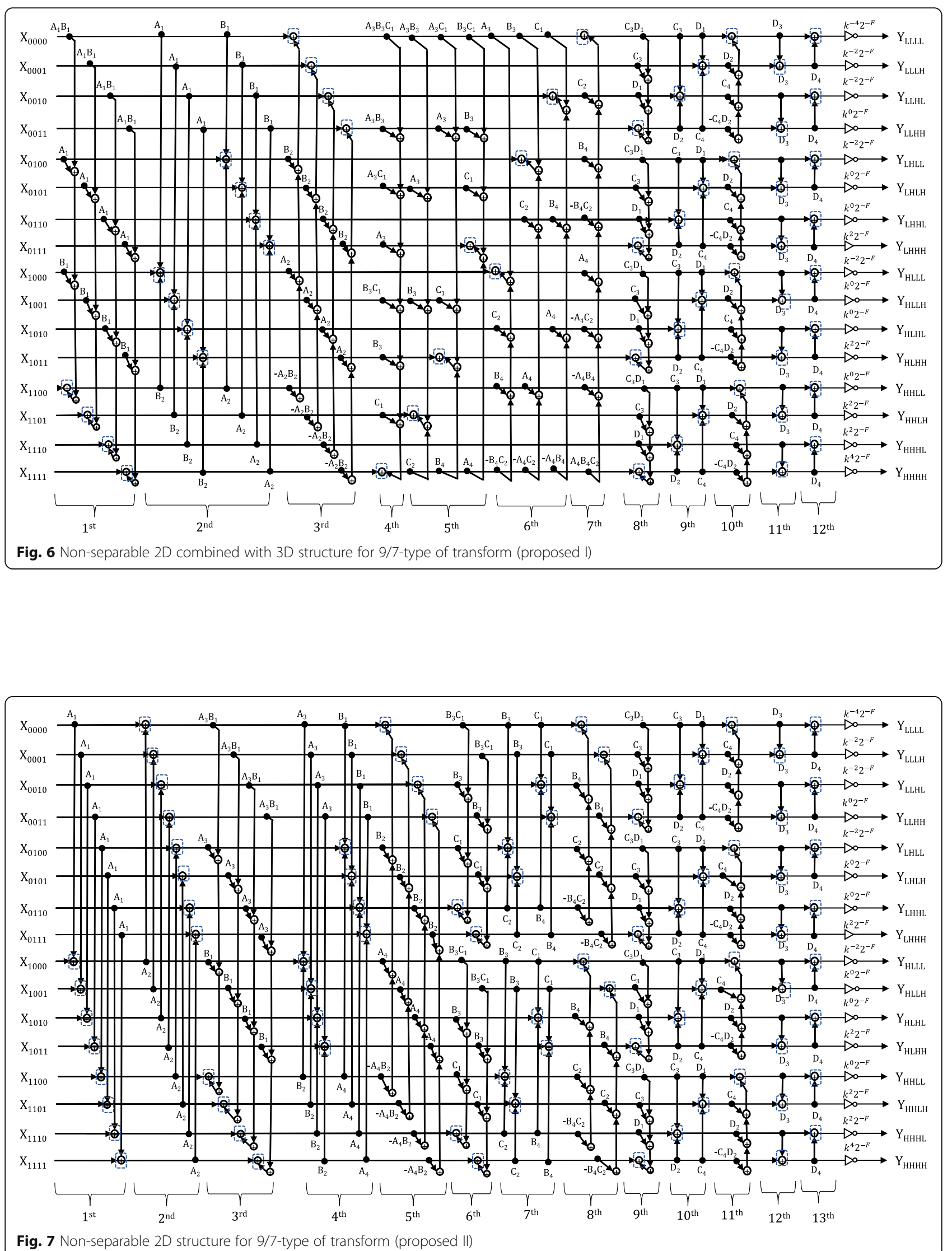


$$
\begin{aligned}
X_{11 c_{1} c_{2}}^{(2)}(\mathbf{z})= & X_{11 c_{1} c_{2}}^{(1)}(\mathbf{z}) \\
& +R\left[A_{3} B_{1} X_{00 c_{1} c_{2}}^{(1)}(\mathbf{z})+A_{3} X_{01 c_{1} c_{2}}^{(1)}(\mathbf{z})+B_{1} X_{10 c_{1} c_{2}}^{(1)}(\mathbf{z})\right], \\
& \text { where } c_{1}, c_{2} \in\{0,1\},
\end{aligned}
$$

Thus, proposed II is a combination of non-separable 2D structures and separable 1D structures.

\subsection{Comparison of the structures}

Table 1 compares the four structures: separable 4D, nonseparable 3D, non-separable $2 \mathrm{D}$ and $3 \mathrm{D}$, and non-separable 2D. As summarized in the table, the total number of lifting steps in the proposed non-separable 2D structure increases from 12 to 13 comparing with the existing non-separable $3 \mathrm{D}$ structure. However, the total number of rounding operators remains the same. Note that both are still smaller in number than the existing separable 4D structure. A different structure obtained by combining the $2 \mathrm{D}$ and $3 \mathrm{D}$ structures is first proposed to further reduce the number of rounding operators, but to reduce rounding noise, it is necessary to maintain the original, separable 4D structure. Therefore, the non-separable 2D structure is proposed based on this original structure.

As shown in Fig. 4, the existing I method is composed of lifting steps $A_{1}, A_{2}, \ldots ., D_{4}$, and is expressed as Separable 4D

$$
A_{1} A_{2} A_{3} A_{4} B_{1} B_{2} B_{3} B_{4} C_{1} C_{2} C_{3} C_{4} D_{1} D_{2} D_{3} D_{4}
$$

In existing I, the order of lifting steps for spatial dimension $x$ remains the same, but those of the steps for dimensions $y$ and $z$, and temporal dimension $t$, change as

Separable 3D'

$$
B_{1} B_{2} C_{1} C_{2} D_{1} D_{2} B_{3} B_{4} C_{3} C_{4} D_{3} D_{4}
$$

Part of this is implemented in the non-separable 3D structure (existing II).

Non-separable 3D

$$
A_{1} A_{2} A_{3} A_{4}\left(B_{1} B_{2} C_{1} C_{2} D_{1} D_{2}\right)_{3 D}\left(B_{3} B_{4} C_{3} C_{4} D_{3} D_{4}\right)_{3 D}
$$

Unlike existing II, the proposed I structure is expressed as.

Table 1 Comparison of the methods

\begin{tabular}{llll}
\hline Structure & Lifting steps & $\begin{array}{l}\text { Rounding } \\
\text { operators }\end{array}$ & $\begin{array}{l}\text { Memory } \\
\text { accessing }\end{array}$ \\
\hline $\begin{array}{l}\text { Separable 4D (existing I) } \\
\begin{array}{l}\text { Non-separable 3D } \\
\text { (existing II) }\end{array}\end{array}$ & 12 & 192 & 1D \\
$\begin{array}{l}\text { Non-separable 2D } \\
\text { and 3D (proposed I) }\end{array}$ & 12 & 96 & 1D and 3D \\
$\begin{array}{l}\text { Non-separable 2D } \\
\text { (proposed II) }\end{array}$ & 13 & 72 & 1D, 2D, and 3D \\
\hline
\end{tabular}

Non-separable $2 D \& 3 D$

$$
\left(A_{1} A_{2} B_{1} B_{2}\right)_{2 D}\left(A_{3} A_{4} B_{3} B_{4} C_{1} C_{2}\right)_{3 D}\left(C_{3} C_{4} D_{1} D_{2}\right)_{2 D} D_{3} D_{4}
$$

To maintain the original structure of existing I, proposed II is expressed as.

Non-separable 2D

$$
A_{1} A_{2}\left(A_{3} A_{4} B_{1} B_{2}\right)_{2 D}\left(B_{3} B_{4} C_{1} C_{2}\right)_{2 D}\left(C_{3} C_{4} D_{1} D_{2}\right)_{2 D} D_{3} D_{4}
$$

Note that the brackets refer to the non-separable structure.

\subsection{Derivation of the structure}

The derivation of the non-separable structure in Figs. 5, 6, and 7 from the separable structure in Fig. 4 is detailed here. We derived it using two the basic properties illustrated in Fig. 8 and as described in [32]. They are denoted by

$$
\begin{array}{cl}
\mathrm{P}_{\mathrm{I}}: & \mathbf{Y}=\mathbf{B A} \cdot \mathbf{X}=\mathbf{A C}_{0} \mathbf{B} \cdot \mathbf{X}, \\
\mathrm{P}_{\mathrm{II}}: & \mathbf{Y}=\mathbf{A B} \cdot \mathbf{X}=\mathbf{B} \mathbf{C}_{1} \mathbf{A} \cdot \mathbf{X},
\end{array}
$$

for

$$
\begin{aligned}
& \mathbf{A}=\left[\begin{array}{lll}
1 & 0 & 0 \\
A & 1 & 0 \\
0 & 0 & 1
\end{array}\right], \mathbf{C}_{0}=\left[\begin{array}{ccc}
1 & 0 & 0 \\
0 & 1 & 0 \\
+A B & 0 & 1
\end{array}\right], \mathbf{X}=\left[\begin{array}{c}
X_{0} \\
X_{1} \\
X_{2}
\end{array}\right], \\
& \mathbf{B}=\left[\begin{array}{lll}
1 & 0 & 0 \\
0 & 1 & 0 \\
0 & B & 1
\end{array}\right], \mathbf{C}_{1}=\left[\begin{array}{ccc}
1 & 0 & 0 \\
0 & 1 & 0 \\
-A B & 0 & 1
\end{array}\right], \mathbf{Y}=\left[\begin{array}{c}
Y_{0} \\
Y_{1} \\
Y_{2}
\end{array}\right],
\end{aligned}
$$

By using both the above properties, the process of derivation of the non-separable structure for 4D WT is clarified. The basic properties for modification to be implemented in the non-separable structure shown in Fig. 8a, b have different lifting procedures but are equivalent as shown in Eqs. (38) and (39). By adding properties I and II to the conventional separable structure, the lifting can be grouped together, thus reducing the numbers of lifting steps and rounding operations in the transform. Therefore, the nonseparable structure can be created systematically to reduce rounding and enhance coding performance.

The process of deriving the non-separable $2 \mathrm{D}$ for double lifting is shown in Fig. 9 based on [32]. Note that the same derivation process is used to obtain the non-separable quadruple $3 \mathrm{D}$ structure in Fig. 5, to combine the nonseparable 2D and 3D structures for quadruple 4D integer WT as in Fig. 6, and to employ the non-separable 2D structure for quadruple 4D integer WT as in Fig. 7.

A clear comparison in terms of the numbers of rounding operators and lifting steps between the separable and non-separable structures is provided in Fig. 10. 


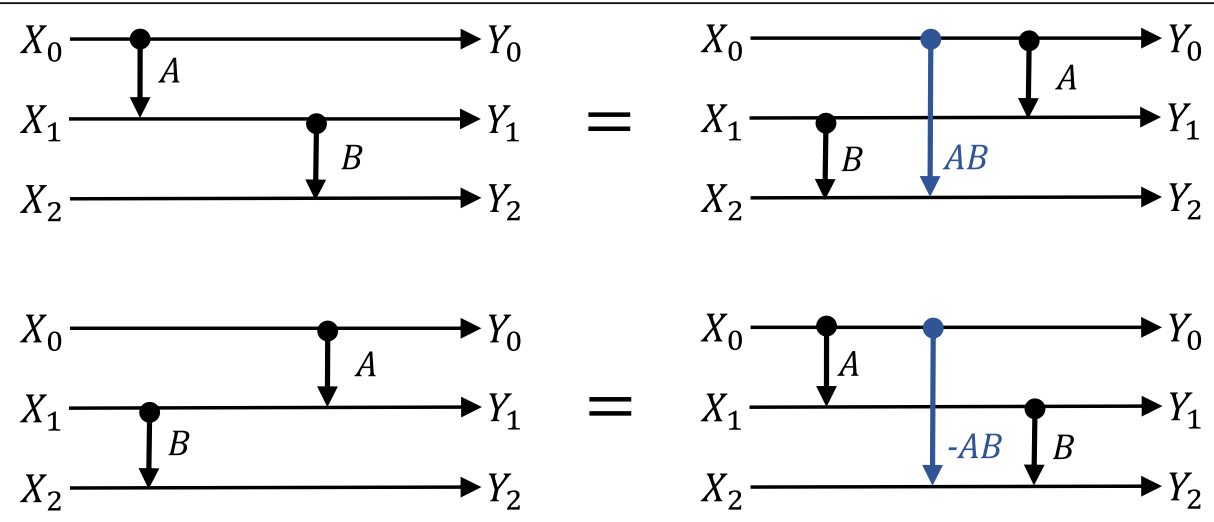

Fig. 8 Basic properties for modification. a Property I. b Property II

\subsection{Lifting steps and latency}

The non-separable structure can reduce the numbers of rounding operations and lifting steps in the transform. The smaller the number of lifting steps, the lower the overall latency of the transform, as shown in [32]. Figure 11a illustrates an example of the implementation of the first lifting step in the doublelifting separable 2D structure shown in Fig. 10a. In this structure, each adder is implemented one by one in a parallel processor with the latency denoted by "A." Similarly, denoting the latency of the multipliers by "M," it takes $M+2 A$ steps in total. Compared with the non-separable 2D structure in Fig. 10b, four adders are simultaneously implemented in a parallel processor for the first and third lifting steps as shown in Fig. $11 \mathrm{~b}$ and take $M+4 A$ steps each. The second lifting step implemented in Fig. $11 \mathrm{c}$ is according to Fig. $10 \mathrm{~b}$ and has $M+3 A$ steps. As a result, the separable 2D structure and the non-separable 2D structure took $4 M+8 A$ and $3 M+11 A$ steps, respectively. Thus, the latency ratio is defined as

$$
L=\frac{3 \eta+11}{4 \eta+8}, \eta=\frac{M}{A}
$$

Owing to the number of lifting steps, latency is reduced if $\eta>3$. Note that the above estimation is valid only for examples shown in Fig. 11. As the non-separable 2D and 3D for quadruple 4D integer WT are designed according to the same derivation of non-separable 2D for double 2D integer WT, the implementation examples in Fig. 11 are applicable to them. The proposed non-separable $2 \mathrm{D}$ for quadruple 4D integer WT is expected to have lower latency than the separable 4D structure.

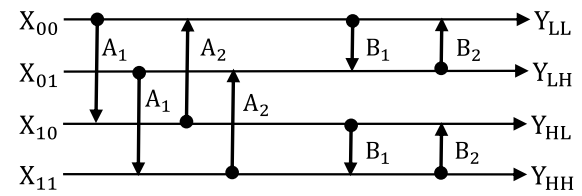

(a) Separable 2D structure

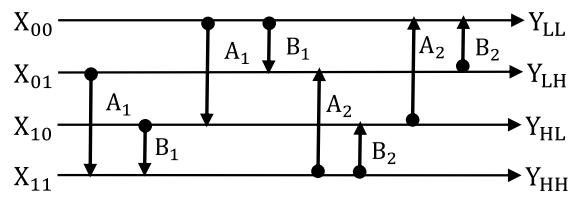

(b) Rearranging the lifting structure

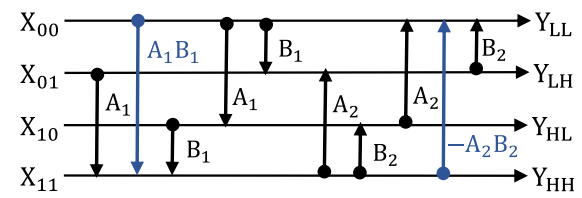

(c) Applying property I and property II

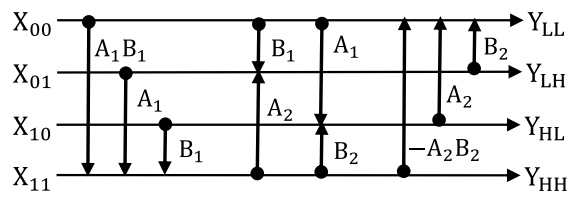

(d) Non-separable 2D structure

Fig. 9 Derivation process for non-separable double-lifting 2D structure. a Separable 2D structure. b Rearranging the lifting structure. c Applying property I and property II. d Non-separable 2D structure 


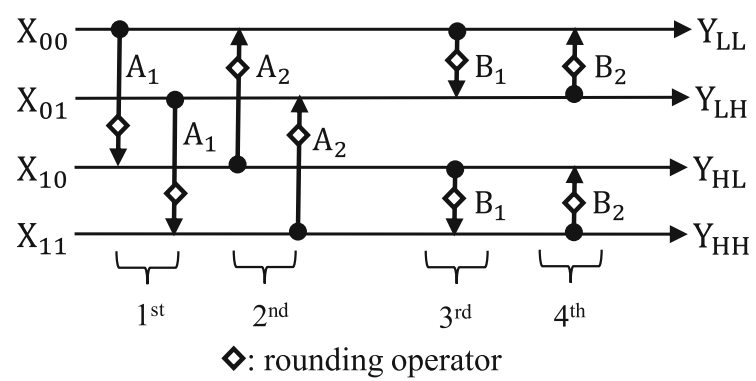

(a) Separable 2D structure for double lifting with 8 rounding operators and 4 lifting steps

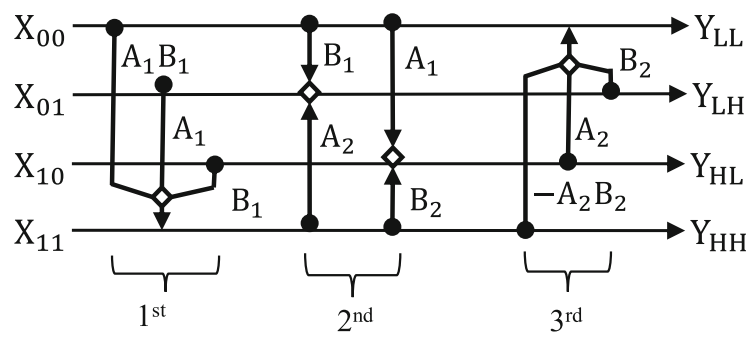

(b) Non-separable 2D structure for double lifting with 4 rounding operators and 3 lifting steps

Fig. 10 Separable and non-separable 2D structures. a Separable 2D structure for double lifting with eight rounding operators and four lifting steps. b Non-separable 2D structure for double lifting with four rounding operators and three lifting steps

\section{Experimental results and discussions}

In the following experiments, six types of data were used to evaluate the rounding noise and coding performance, as shown in Table 2.

Note that the MRI, functional magnetic resonance image (fMRI)(II), and US data were retrieved from [33, 34] and [35], respectively. MRI data represent highly correlated data at consecutive time points with limited motion in the temporal dimension $t$ and structural changes in the spatial dimension $z$.

Each image is normalized to the range $[0,255]$ for display purposes as shown in Fig. 12a-f. In this paper, the variance in noise in frequency domain and coding performance in lossless and lossy coding modes were investigated.

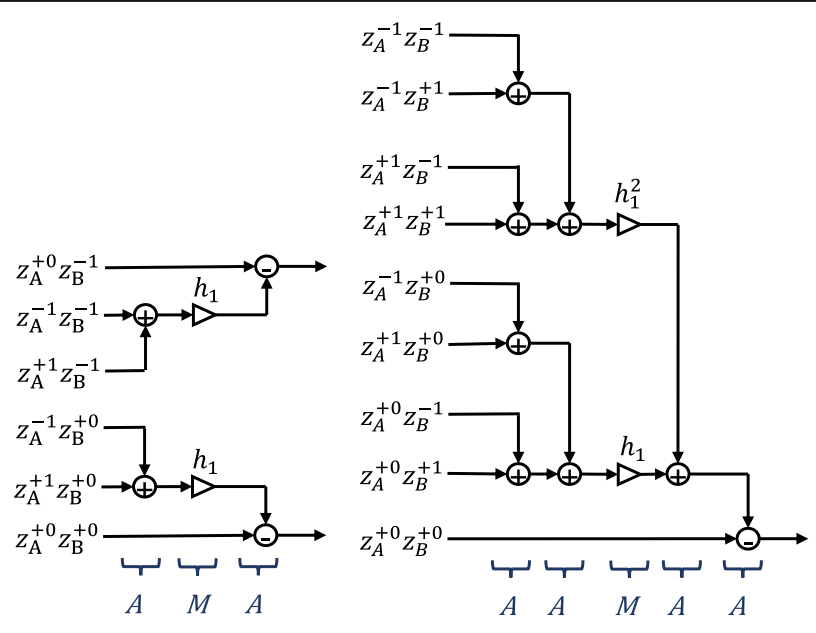

(a) $1^{\text {st }}$ in Fig. 10(a)

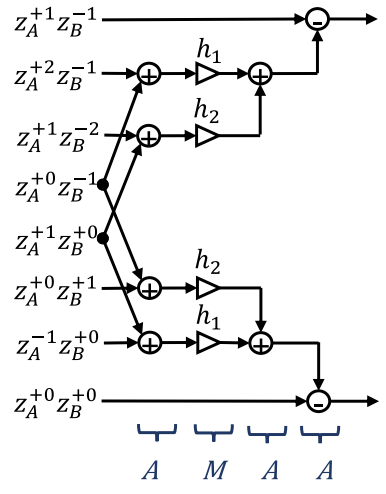

(c) $2^{\text {nd }}$ in Fig. $10(\mathrm{~b})$

Fig. 11 Implementation examples in parallel processing platform 
Table 2 Type of data used in the experiments

\begin{tabular}{|c|c|c|c|}
\hline Type of data & $\begin{array}{l}\text { Size of data in } \\
(x \times y \times z \times t) \\
\text { sequence }\end{array}$ & Bit depth & $\begin{array}{l}\text { Referred } \\
\text { here as }\end{array}$ \\
\hline $\begin{array}{l}\text { 4D functional magnetic } \\
\text { resonance image }\end{array}$ & $56 \times 88 \times 32 \times 16$ & 16 & fMRI (I) \\
\hline $\begin{array}{l}4 \mathrm{D} \text { computed } \\
\text { tomography image }\end{array}$ & $256 \times 256 \times 16 \times 16$ & 12 & $\mathrm{CT}$ \\
\hline $\begin{array}{l}\text { 4D functional magnetic } \\
\text { resonance image }\end{array}$ & $40 \times 64 \times 64 \times 16$ & 12 & fMRI (II) \\
\hline $\begin{array}{l}4 \mathrm{D} \text { magnetic } \\
\text { resonance image }\end{array}$ & $52 \times 224 \times 64 \times 16$ & 8 & MRI \\
\hline $\begin{array}{l}\text { 4D auto-regressive } \\
\text { model }\end{array}$ & $256 \times 256 \times 32 \times 16$ & 8 & $A R$ \\
\hline 4D ultrasound image & $532 \times 416 \times 16 \times 16$ & 8 & US \\
\hline
\end{tabular}

The 4D auto-regressive (AR) model used in our experiments can be expressed as

$$
\left\{\begin{array}{c}
x^{(1)}\left(n_{1}, n_{2}, n_{3}, n_{4}\right)=x\left(n_{1}, n_{2}, n_{3}, n_{4}\right)+\rho \cdot x^{(1)}\left(n_{1}-1, n_{2}, n_{3}, n_{4}\right) \\
x^{(2)}\left(n_{1}, n_{2}, n_{3}, n_{4}\right)=x^{(1)}\left(n_{1}, n_{2}, n_{3}, n_{4}\right)+\rho \cdot x^{(2)}\left(n_{1}, n_{2}-1, n_{3}, n_{4}\right) \\
x^{(3)}\left(n_{1}, n_{2}, n_{3}, n_{4}\right)=x^{(2)}\left(n_{1}, n_{2}, n_{3}, n_{4}\right)+\rho \cdot x^{(3)}\left(n_{1}, n_{2}, n_{3}-1, n_{4}\right) \\
x^{(4)}\left(n_{1}, n_{2}, n_{3}, n_{4}\right)=x^{(3)}\left(n_{1}, n_{2}, n_{3}, n_{4}\right)+\rho \cdot x^{(4)}\left(n_{1}, n_{2}, n_{3}, n_{4}-1\right)
\end{array}\right.
$$

Note that $\rho$ was set to 0.9 in the experiments in this paper, as the typical values of $\rho$ for natural images are between 0.9 and 0.98 [36].

\subsection{Evaluation of rounding noise}

As fewer rounding operators do not imply fewer rounding errors in total $[21,22]$, the total rounding noise in the output frequency band signal was investigated. The rounding operation is a vital part of lossy compression.

A non-linear operation transforms a floating-point signal into an integer signal. An equivalent expression to the rounding operation is shown in Fig. 13. A non-linear equation with additive noise is defined as

$$
S_{R_{o}}=S_{R_{i}}(z)+N_{R}(z),
$$

where $S_{R_{i}}, S_{R_{o}}$, and $N_{R}$ denote the input signal, the output signal, and the additive noise of the rounding operation, respectively. As the correlation between each of the of the errors and the signals was zero (based on statistical independence), the variance of output signal was calculated from

$$
\sigma_{S_{R_{o}}}^{2}=\sigma_{S_{R_{i}}}^{2}+\sigma_{N_{R}}^{2}
$$

where $\sigma_{S_{R_{j}}}^{2}, \sigma_{S_{R_{o}}}^{2}$, and $\sigma_{N_{R}}^{2}$ refer to the variance of the input signal, the output signal, and the additive noise of the rounding operators, respectively. As the power of the probability density function (PDF) of additive noise is approximately flat, as shown in Fig. 14, the variance of the additive noise for the rounding operations was calculated as

$$
\sigma_{N_{R}}^{2}=\int_{-0.5}^{0.5} x^{2} d x=\frac{1}{12},
$$

In this study, rounding noise inside the circuit was measured to observe the accumulative error in it due to the rounding operators. Figure 15 illustrates that rounding

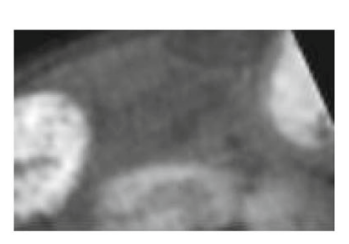

(a) fMRI (I)

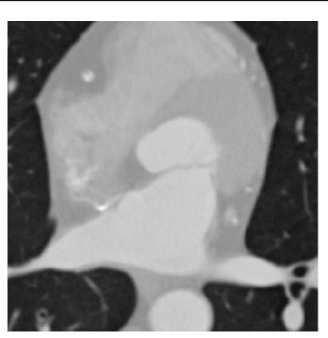

(b) $\mathrm{CT}$

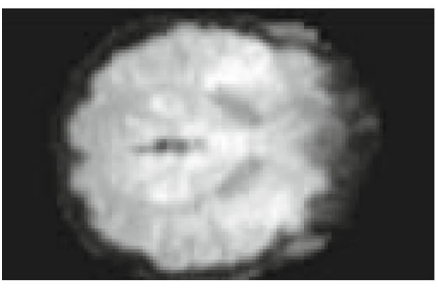

(d) fMRI (II)

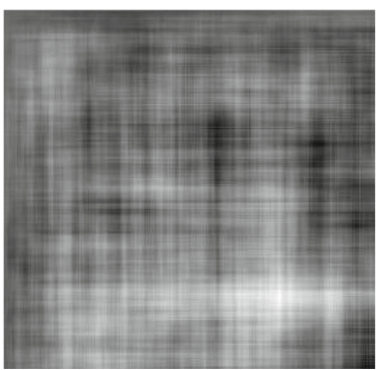

(e) AR

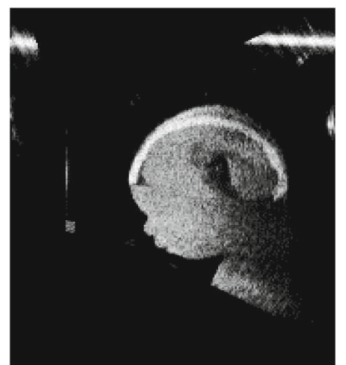

(f) US

Fig. 12 Tested data. a fMRI (I). b CT. c MRI. d fMRI (II). e AR. f US 


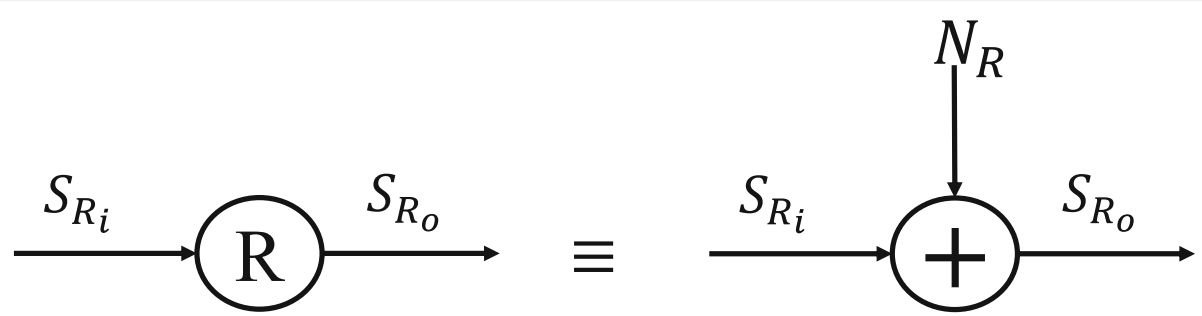

Fig. 13 Rounding operation and its equivalent expression

noise was examined by using the error between the integer signal and the real number signal. Rounding noise is defined by Eq. (47).

$$
\text { error }=y-\widehat{y}
$$

Rounding noise was evaluated in six types of input signals to compare the conventional and the proposed lifting structures, as shown in Fig. 16a-f.

Figure $16 \mathrm{a}-\mathrm{f}$ indicate the variance of the rounding error in each frequency band for fMRI(I), CT, fMRI(II), MRI, AR, and US data respectively. The average magnitude of rounding errors for all data was reduced to 19 . $11,16.08,16.81,5.63$, and $16.09 \%$, respectively, except for US data, which was increased by $15.47 \%$, between existing I structure and proposed II structure, as shown in Fig. 17. However, as the rounding noise in the LLLL frequency band for proposed II structure for the US data was lower than that in existing I, the coding performance on the US data for proposed II method improved as shown in Fig. 20f. The HHHH frequency band of the existing II structure was the highest and resulted in a degradation in coding performance. The energy of the frequency band should be compacted to a low-frequency band signal to reduce entropy in the compressed image and enhanced coding performance. The higher the variance of rounding noise in the low-frequency band signal, the lower the entropy of the compressed image. However, all structures had the highest compacted energy in the high-frequency band signals. Therefore, we conclude that the lowest variance in rounding noise among all frequency band signals yields the best coding performance.

The reason for why the total number of rounding operators did not influence total rounding noise is investigated in Fig. 18, which shows the variance of rounding noise in the frequency domain. In an experiment, only one rounding operator in the forward transform was activated. The horizontal axis denotes the activated rounding operator, numbered from top to bottom, from left to right in Figs. 4 to 7 . As summarized in Table 1, the existing I, existing II, proposed I, and proposed II structures had 192, 96, 72, and 96 rounding operations, respectively. The total rounding noise in proposed II decreased even though the number of rounding operations, the source of the noise, remained the same as that in the existing II structure. This is because of the relatively large variance in error in the structure. The variance in rounding noise was the highest in the 63rd rounding operator at 0.1844 , the ninth rounding operator at 1 . 2217 , the 14th rounding operator at 0.5964 , and the 56th rounding operator at 0.2127 for existing I,

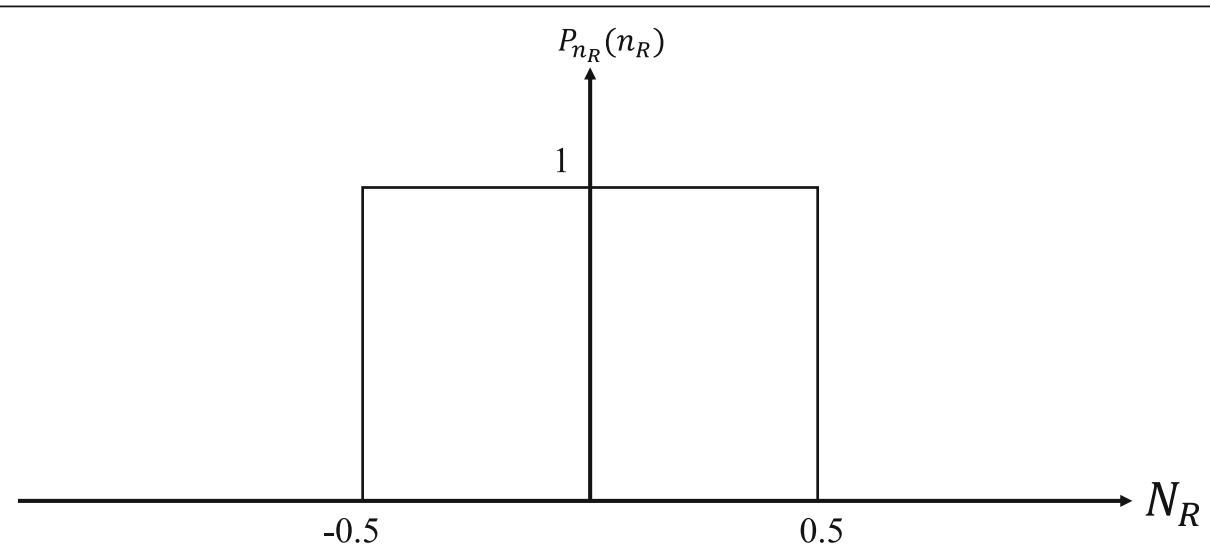

Fig. 14 Probability density function (PDF) for an amplitude of the additive noise 


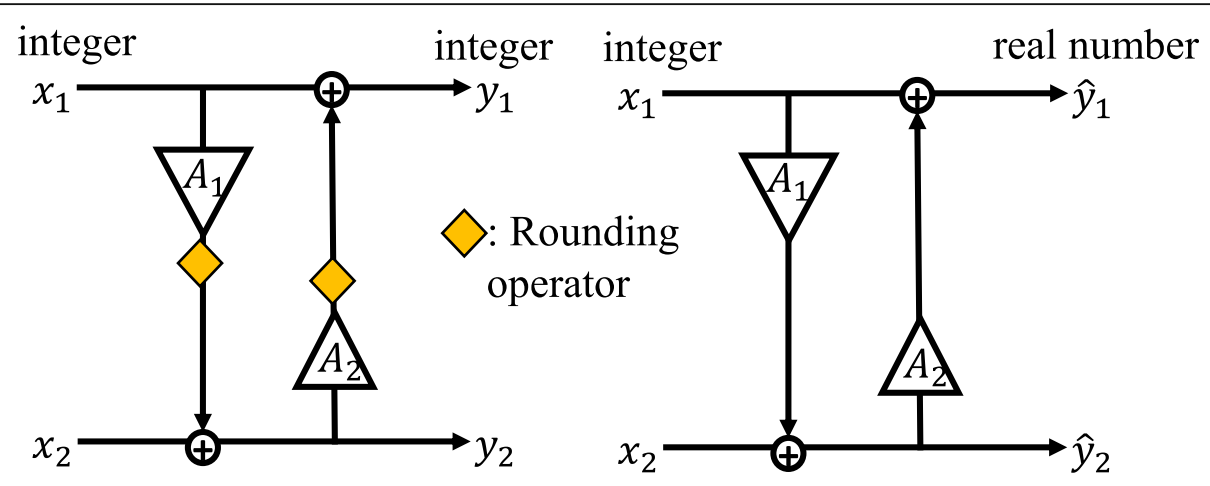

Fig. 15 Rounding noise between integer and real number

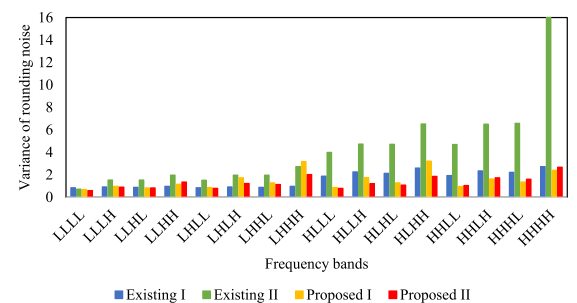

(a) fMRI(I)

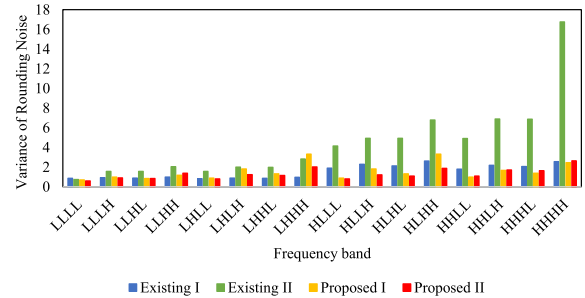

(b) $\mathrm{CT}$

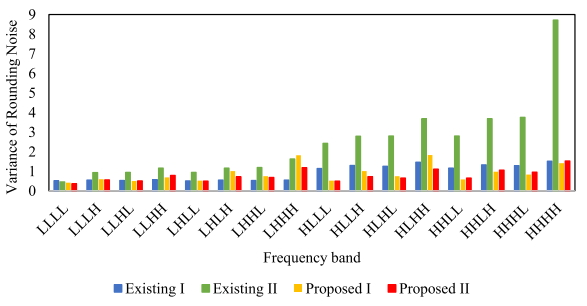

(c) fMRI(II)

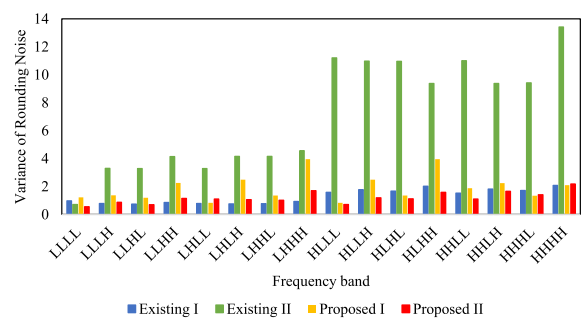

(d) MRI

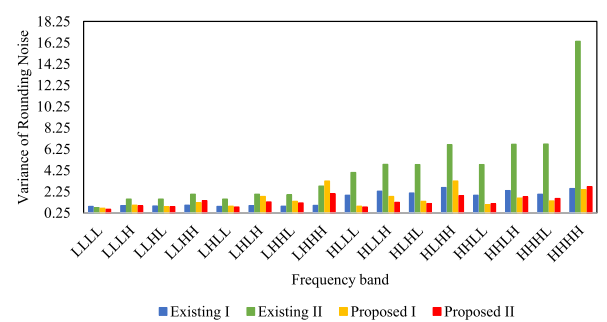

(e) AR

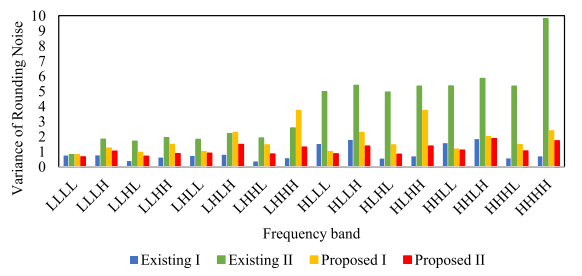

(f) US

Fig. 16 Variance of rounding noise for all data in each frequency band. a fMRI(I). b CT. c fMRI(II). d MRI. e AR. f US 


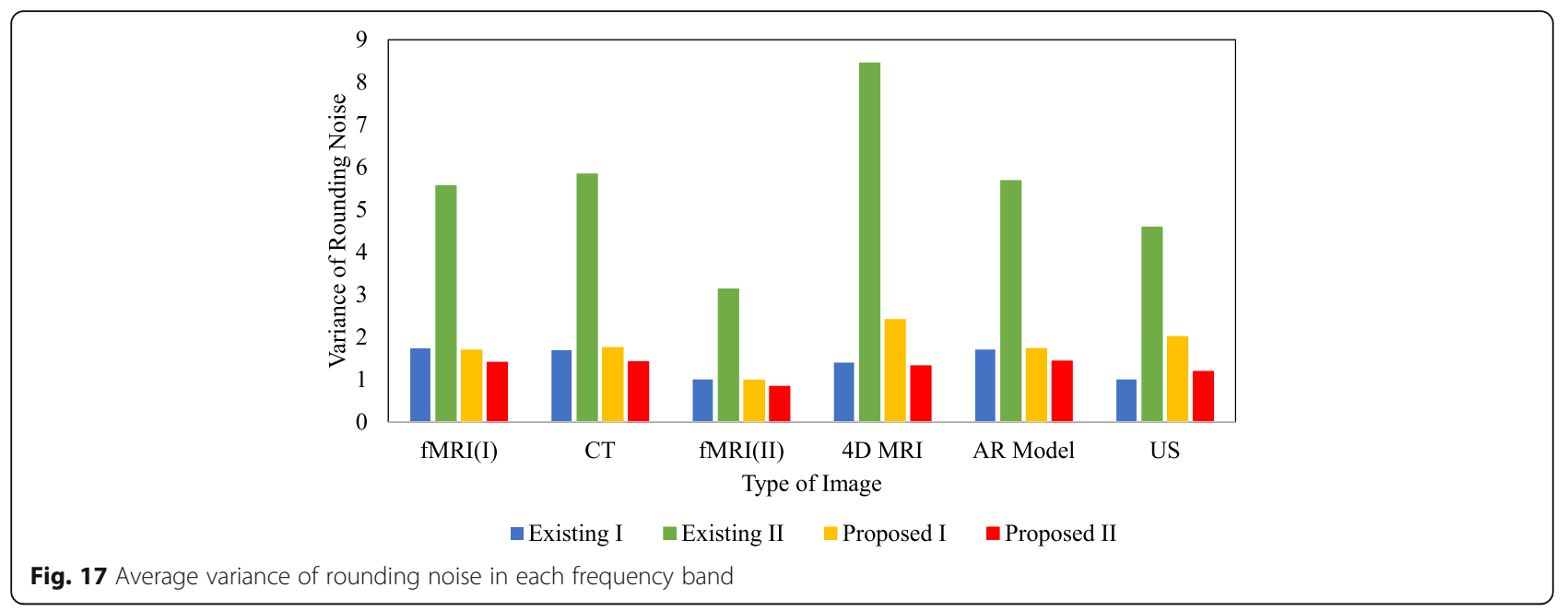

existing II, proposed I, and proposed II, respectively. However, the average rounding noise in proposed II relative to existing I decreased by almost half from 0 . 0646 to 0.0382 . It can be concluded that the more frequent and the greater the extent to which noise is amplified in the lifting structure, the higher the rounding noise inside it. As the rounding noise inside the transform influences coding performance, it is investigated in Section 5.2.

\subsection{Evaluation of coding performance}

Figure 19 illustrates the rate distortion curve, which compares the performance of the methods in the lossy coding mode for CT images. The horizontal and vertical axes represent the entropy rate measured in bits per pixel (bpp) and the peak signal-to-noise ratio (PSNR) of the reconstructed signal, respectively. It shows that the proposed II structure outperformed the others at the same bitrate. The quality of the reconstructed signal in proposed II increased by $0.18,16.11$, and $26.44 \mathrm{~dB}$ over existing I, existing II, and proposed I, respectively. As the quality of the reconstructed image deteriorated considerably if it was transformed using existing II and proposed I. Figure 20a-e shows the coding performance results when images were transformed using existing I and proposed II only.

Figure 20a-e shows the coding performance of existing I and proposed II on fMRI(I), fMRI(II), MRI, AR, and US data, respectively. Under the same bitrate, the quality of the reconstructed signal for proposed II increased by 0.40 , 2.10, $0.27,2.57$, and $0.72 \mathrm{~dB}$ for fMRI(I), fMRI(II), MRI, $\mathrm{AR}$, and US data, respectively. As both quantization noise

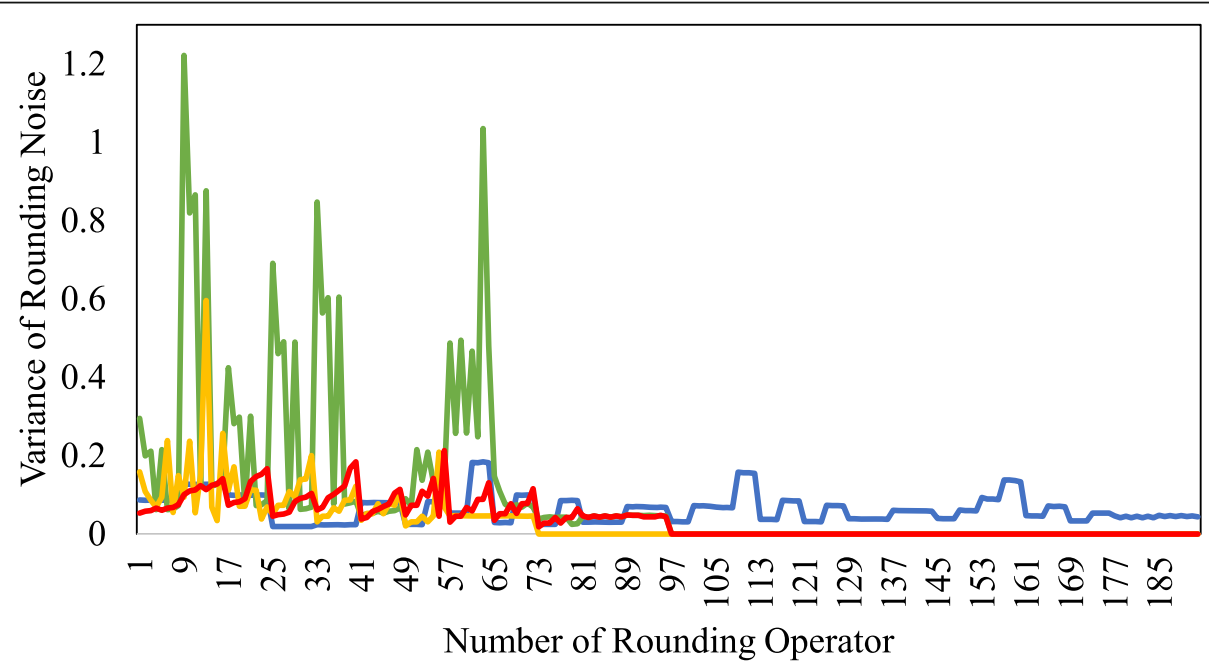

—Existing I — Existing II - Proposed I —Proposed II

Fig. 18 Effect of one rounding operator for fMRI(II) data 


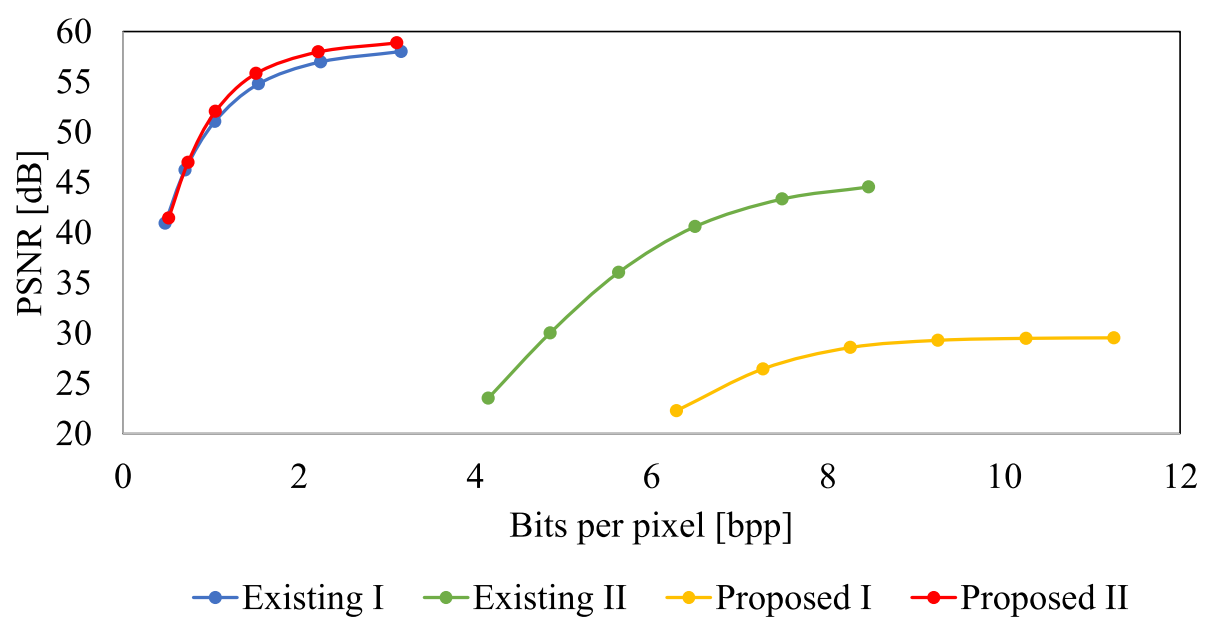

Fig. 19 Coding performance in lossy mode for CT image

and rounding noise were present in the lossy compression system, even though the former for all structures was the same, rounding noise in the proposed II structure was the lowest. Thus, the coding performance of this structure was the best. In conclusion, proposed II structure outperformed all other structures in the experiments.

\section{Conclusions}

This paper proposed a non-separable 2D structure for $4 \mathrm{D}$ input signals in lieu of non-separable 3D structures. The total number of rounding operators was reduced by half compared with the prevalent separable 4D structure. However, rounding noise owing to the integer implementation of signal values inside the transform increased in the nonseparable 3D structure, due to a change in its original lifting scheme. Therefore, by maintaining the original scheme, the proposed non-separable 2D structure reduced total rounding noise in it and enhanced the quality of the reconstructed signal in lossy coding. Furthermore, the number of lifting steps, a reduction in which reduces the latency of the overall transform, was also reduced by $18.75 \%$ between the proposed non-separable 2D structure and the conventional separable 4D structure on the quadruple 4D integer WT. Our integer WT has the advantage of compatibility with the conventional integer WT. It also enhances compression performance on $4 \mathrm{D}$ images, such as medical images.

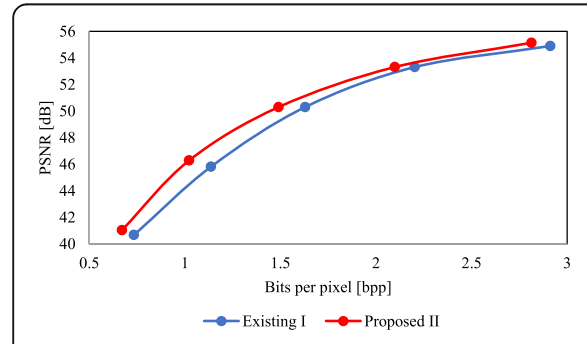

(a) $\operatorname{fMRI}(\mathrm{I})$

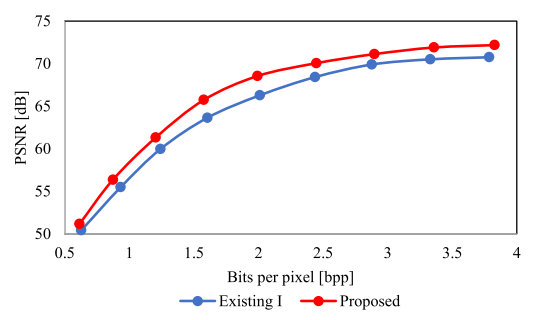

(b) fMRI(II)

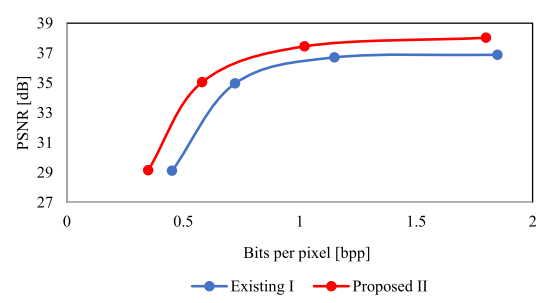

(c) MRI

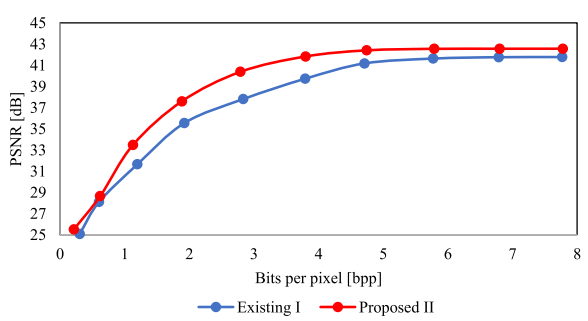

(d) AR

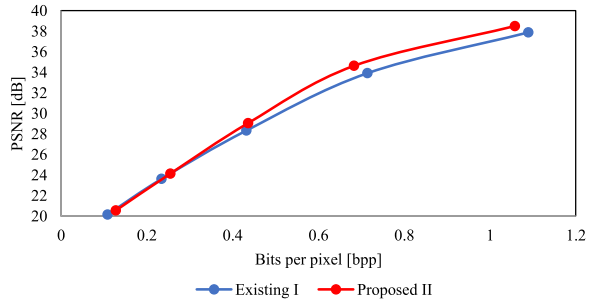

(e) US

Fig. 20 Coding performance in lossy mode for $f M R I(I)$, fMRI(II), MRI, AR, and US data. a fMRI(I). b fMRI(II). c MRI. d AR. e US 


\section{Abbreviations}

1D: One-dimensional; 2D: Two-dimensional; 3D: Three-dimensional; 4D: Fourdimensional; AR: Auto-regressive; Bpp: Bits per pixel; CT: Computed Tomography; DCT: Discrete cosine transform; DWT: Discrete wavelet transform; fMRI: Functional magnetic resonance image; JPEG: Joint photographic experts group; MRI: Magnetic resonance imaging; PDF: Probability density function; PSNR: Peak signal-to-noise ratio US: Ultrasound; WT: Wavelet transform

\section{Acknowledgements}

We thank Saad Anis, Ph.D, from the Edanz Group (www.edanzediting.com/ac) for editing a draft of this manuscript.

\section{About the authors}

Fairoza Amira Binti Hamzah received the Diploma of Electrical and Electronics Engineering from the Japanese Associate Degree Program, Selangor Industrial University, Malaysia, in 2012. Then, she received B. Eng. and M. Engrg. degree in Electrical, Electronics and Information Engineering in Nagaoka University of Technology in 2014 and 2016, respectively, and is currently pursuing Ph.D. of Engineering in the Information Science and Control Engineering department of the same university. In 2017, she had a research internship experience in Department of Computer Science, University of Warwick, UK. Her research interests are in digital signal processing and image compression. She is a Graduate Member of Board of Engineers Malaysia (BEM) and the Institute of Engineers Malaysia (IEM), and a Graduate Student Member of IEEE.

Sayaka Minewaki received her B. Eng. and M. Eng. degrees in Engineering from Kyushu Institute of Technology in 2001 and 2003, respectively. In 2006, she finished a Ph.D. program without dissertation at the Department of Artificial Intelligence, Kyushu Institute of Technology. In 2006, she joined the Yuge National College of Technology, where she served concurrently as a Lecturer. In 2016, she joined Nagaoka University of Technology, where she is currently an Assistant Professor of the Department of Electrical, Electronics and Information Engineering. Her research interests are in the fields of digital signal processing, image compression and natural language processing. Taichi Yoshida received B. Eng., M.Eng., and Ph.D. degrees in Engineering from Keio University, Yokohama, Japan, in 2006, 2008, and 2013, respectively. In 2014, he joined Nagaoka University of Technology as an Assistant Professor in the Department of Electrical, Electronics and Information Engineering. He then joined the University of Electro-Communications, Tokyo, Japan in 2018. His research interests are in the field of filter bank design and image coding application. He is a member of IEEE. Masahiro Iwahashi received his B. Eng, M. Eng., and D. Eng. degrees in electrical engineering from Tokyo Metropolitan University in 1988, 1990, and 1996, respectively. In 1990, he joined Nippon Steel Co. Ltd. From 1991 to 1992, he was dispatched to Graphics Communication Technology Co. Ltd. In 1993, he joined Nagaoka University of Technology, where he is currently a professor of the Department of Electrical Engineering, Faculty of Technology. From 1995 to 2001, he served concurrently as a lecturer of Nagaoka Technical College. From 1998 to 1999, he was dispatched to Thammasat University in Bangkok, Thailand, as a JICA expert. His research interests are in the area of digital signal processing, multi-rate systems, and image compression. From 2007 to 2011, he served as an editorial committee member of the transaction on fundamentals. He is serving as a reviewer of IEEE, IEICE, and APSIPA. He is currently a senior member of the IEEE and IEICE.

\section{Availability of data and materials}

Please contact author for data requests.

\section{Authors' contributions}

The corresponding and first author is the person who made the substantial contributions to the conception and design, acquisition, analysis and interpretation of the data. The manuscript is critically revised for intellectual content by the second, third, and fourth authors.

\section{Ethics approval and consent to participate}

Not applicable.

\section{Competing interests}

The authors declare that they have no competing interests.

\section{Publisher's Note}

Springer Nature remains neutral with regard to jurisdictional claims in published maps and institutional affiliations.

\section{Author details}

${ }^{1}$ Department of Electrical, Electronics and Information Engineering, Nagaoka University of Technology, Niigata, Japan. ${ }^{2}$ Department of Computer and Network Engineering, The University of Electro-Communications, Tokyo, Japan.

Received: 13 July 2017 Accepted: 24 April 2018

Published online: 28 May 2018

References

1. M Unser, T Blu, Mathematical properties of the JPEG 2000 wavelet filters. IEEE Trans. Image Process. 12(9), 1080-1090 (2003)

2. M Antonini, M Barlaud, P Mathieu, I Daubechies, Image coding using wavelet transforms. IEEE Trans. Image Process. 1(2), 205-220 (1992)

3. D Le Gall, A Tabatai, in International Conference on Acoustics, Speech and Signal Processing. Subband coding of digital images by using symmetric short kernel filters and arithmetic coding techniques (1988)

4. W Sweldens, The lifting scheme: a custom design construction of biorthogonal wavelets. Appl. Comput. Harmon. Analysis 3(2), 186-200 (1996)

5. A Nait-Ali, C Cavaro-Menard, Compression of Biomedical Images and Signals (Wiley, USA, 2008)

6. HK Huang, PACS and Imaging Informatics: Basic Principles and Applications (Wiley, USA, 2010)

7. R Rajeswari, R Rajesh, in World Congress on Nature \& Biologically Inspired Computing. Efficient compression of 4D fMRI images using Bandelet transform and fuzzy thresholding (IEEE, India, 2009).

8. V Sanchez, P Nasiopoulos, R Abughrabieh, Novel lossless fMRI image compression based on motion compensation and customized entropy coding. IEEE Trans. Inf Techno Biomed 13(4), 645-655 (2009)

9. HG Lalgudi, A Bilgin, MW Marcellin, A Tabesh, MS Nadar, TP Trouard, Fourdimensional compression of fMRI using JPEG 2000. Proc. of SPIE, Medical Imaging: Image Processing 5747, 1028-1037 (2005)

10. C Chrysafis, A Ortega, Line-based, reduced memory, wavelet image compression. IEEE Trans. Image Process. 9(3), 378-389 (2000)

11. G Shi, W Liu, L Zhang, F Li, An efficient folded architecture for lifting-based discrete wavelet transform. IEEE Trans. Circuits, Systems II express briefs 56(4), 290-294 (2009)

12. M Vetterli, C Herley, Wavelets and filter banks: theory and design. IEEE Trans. Signal Processing 40(9), 2207-2232 (1992)

13. DS Taubman, in IEEE International Conference on Image Processing. Adaptive, non-separable lifting transforms for image compression (1999)

14. S Fukuma, M Iwahashi, N Kambayashi, in IEEE International Symposium on Circuits and Systems. Adaptive multi-channel prediction for lossless scalable coding (1999)

15. C Yan, Y Zhang, J Xu, F Dai, L Li, Q Dai, F Wu, A highly parallel framework for HEVC coding unit partitioning tree decision on many-core processors. IEEE Signal Process Lett 21(5), 573-576 (2014)

16. J Franco, G Bernabe, J Fernandez, ME Acacio, in Euromicro Int. Conf. On Parallel, Distributed and Network-Based Processing. A parallel implementation of the 2D wavelet transform using CUDA (IEEE, Germany, 2009)

17. T Yoshida, T Suzuki, S Kiyochi, M Ikehara, Two dimensional non-separable adaptive directional lifting structure of discrete wavelet transform. IEICE Trans. Fundam E94-A(10), 1920-1927 (2011)

18. T Bruylants, A Munteanu, P Schelkens, Wavelet based volumetric medical image compression. Signal Process. Image Commun. 31, 112-133 (2015)

19. FA Binti Hamzah, T Yoshida, M Iwahashi, H Kiya, Adaptive directional lifting structure of three dimensional non-separable discrete wavelet transform for high resolution volumetric data compression. IEICE Trans. Fundam E99-A(5), 892-899 (2016)

20. M Iwahashi, H Kiya, in IEEE International Conference Image Processing. Non separable 2D factorization of separable 2D DWT for lossless image coding (2009)

21. T Strutz, I Rennert, Two-dimensional integer wavelet transform with reduced influence of rounding operations. EURASIP J Advances Signal Process 75 (2012). https://doi.org/10.1186/1687-6180-2012-75

22. M Iwahashi, H Kiya, in Discrete Wavelet Transforms - a Compendium of New Approaches and Recent Applications. Discrete wavelet transforms: non separable two dimensional discrete wavelet transform for image signals 
(InTechOpen, 2013). Available from: https://www.intechopen.com/books/ discrete-wavelet-transforms-a-compendium-of-new-approaches-and-recentapplications/non-separable-two-dimensional-discrete-wavelet-transform-forimage-signals

23. Y Wang, H Hamza, in Industrial Engineering Research Conference. $4 D$ geometry compression based on lifting wavelet transform (The Institute for Operations Research and the Management Sciences, Florida, 2006)

24. JM Gomez, JB Rapesta, I Blanes, LJ Rodriguez, FA Llinas, JS Sagrista, "4D remote sensing image coding with JPEG2000," Satellite Data Compression, Communications and Processing VI, vol. 7810, 2010. https://doi.org/10.1117/ 12.860545

25. C-L Kuo, Y-Y Lin, Y-C Lu, in IEEE Int. SOC Conference. Analysis and implementation of discrete wavelet transform for compressing fourdimensional light field data (IEEE, Germany, 2013)

26. A Sang, T Sun, H Chen, H Feng, in Int. Conf. On Image Analysis and Signal Processing. A 4D nth-order Walsh orthogonal transform algorithm used for color image coding (IEEE, China, 2010)

27. M Iwahashi, T Orachon, H Kiya, in IEEE International Conference on Image Processing. Three dimensional discrete wavelet transform with deduced number of lifting steps (2013)

28. M Iwahashi, T Orachon, H Kiya, in Proc. of Asia Pacific Signal and Information Processing (APSIPA) Annual Summit and Conference (ASC). Non separable 3D lifting structure compatible with separable quadruple lifting DWT (2013)

29. FA Binti Hamzah, T Yoshida, M Iwahashi, in Proc. of IEEE ICASSP. Nonseparable Quaruple lifting structure for four-dimensional integer wavelet transform with reduced rounding noise (IEEE, New Orleans, 2017)

30. M Iwahashi, H Kiya, in Discrete Wavelet Transforms. Condition on word length of signals and coefficients for DC lossless property (InTechOpen, ISBN 978-953-307-313-2, 2011), pp. 231-254. Available from: https://www. intechopen.com/books/discrete-wavelet-transforms-algorithms-andapplications/condition-on-word-length-of-signals-and-coefficients-for-dclossless-property-of-dwt

31. "Joint Photographic Experts Group: JPEG 2000 Image Coding System". Patent ISO / IEC FCD 15444-1, 2000

32. S Poomrittigul, M Iwahashi, H Kiya, Reduction of lifting steps of non separable 2D quadruple lifting DWT compatible with separable 2D DWT. IEICE Trans. Fundam. Electron. Commun. Comput. Sci. E97-A(7), 1492-1499 (2014)

33. D Boye et al., in Proc. SPIE 8669, Medical Imaging 2013: Image Processing. Population based modeling of respiratory lung motion and prediction from partial information (2013)

34. JV Haxby, MI Gobbini, ML Furey, A Ishai, JL Schouten, P Pietrini, Distributed and overlapping representations of faces and objects in ventral temporal cortex. Science 293(5539), 2425-2430 (2001)

35. C Cortes, L Kabongo, I Macia, OE Ruiz, J Florez, Ultrasound image dataset for image analysis algorithms evaluation, Innovation in Medicine and Healthcare 2015. Smart Innovation, Systems and Technologies, vol 45 (2015), pp. 447-457

36. J-R Ohm, in Multimedia communication technology: representation, transmission and identification of multimedia signals. Linear systems and transforms (Springer-Verlag, New York, 2004), pp. 96-104

\section{Submit your manuscript to a SpringerOpen ${ }^{\circ}$ journal and benefit from:}

- Convenient online submission

- Rigorous peer review

- Open access: articles freely available online

- High visibility within the field

- Retaining the copyright to your article

Submit your next manuscript at $>$ springeropen.com 